\title{
Parent Functioning and Child Psychotherapy Outcomes: Predicting Outcomes in Usual Care
}

Anna Elise Packard

Brigham Young University - Provo

Follow this and additional works at: https://scholarsarchive.byu.edu/etd

Part of the Psychology Commons

\section{BYU ScholarsArchive Citation}

Packard, Anna Elise, "Parent Functioning and Child Psychotherapy Outcomes: Predicting Outcomes in Usual Care" (2009). Theses and Dissertations. 2311.

https://scholarsarchive.byu.edu/etd/2311

This Dissertation is brought to you for free and open access by BYU ScholarsArchive. It has been accepted for inclusion in Theses and Dissertations by an authorized administrator of BYU ScholarsArchive. For more information, please contact scholarsarchive@byu.edu, ellen_amatangelo@byu.edu. 
Parent Functioning and Child Psychotherapy Outcomes:

Predicting Outcomes in Usual Care

Anna E. Packard

A dissertation submitted to the faculty of

Brigham Young University

in partial fulfillment of the requirements for the degree of

Doctor of Philosophy

Jared S. Warren, Chair

Gary M. Burlingame

Michael J. Lambert

Sally H. Barlow

Joseph A. Olsen

Department of Psychology

Brigham Young University

August 2010

Copyright ( 2009 Anna E. Packard

All Rights Reserved 


\author{
ABSTRACT \\ Parent Functioning and Child Psychotherapy Outcomes: \\ Predicting Outcomes in Usual Care
}

Anna E. Packard

Department of Psychology

Doctor of Philosophy

A prominent need in the youth psychotherapy literature includes the examination of mechanisms of change within the context of "real world" clinical settings, where the practice of psychotherapy differs significantly from that in controlled clinical trials. In examining mechanisms of change in youth psychotherapy, variables related to parent functioning may be among the most important factors to consider in predicting and promoting good child outcomes. The purpose of the present study was to evaluate three important aspects of parent functioningpsychological symptom distress, interpersonal relations, and social role performance-as potential predictors of successful treatment outcomes in a traditional community outpatient treatment setting for children and adolescents. Further, this study examined whether parents indirectly benefited from their children receiving services, expanding our view on the scope and benefits inherent in youth psychotherapy. Parent Symptom Distress, Interpersonal Relations, and Social Role performance were measured using the domains of the Outcome Questionnaire 45 (OQ-45; Lambert et al., 2004), and youth treatment outcomes were measured using the parent and self-report versions of the Youth-Outcome Questionnaire (Y-OQ; Burlingame, Wells, Lambert, \& Cox, 2004; Y-OQ-SR; Wells, Burlingame \& Rose, 2003). Using Hierarchical Linear Modeling with this sample of 339 youth, aged 4-17 and their parents, this study examined the relationship between these parent domains and youth progress in therapy. Results revealed that parent Symptom Distress and Social Role performance improved significantly over the course of youth treatment. Further, Social Role performance at intake significantly predicted the rate of change in parent-reported youth outcome; and Interpersonal Relations at intake significantly predicted rate of change in youth-reported outcome. Finally, changes in parent Social Role performance were associated with changes in youth symptoms over the course of treatment. Examining the associations between these variables is an important step toward identifying potential mechanisms of change in youth mental health treatment. The results of this study provide valuable information on the importance of attending to parent functioning in the assessment and treatment of youth mental health issues.

Keywords: youth psychotherapy, treatment outcome, parent distress, mental health services research, usual care, Youth Outcome Questionnaire 


\section{ACKNOWLEDGEMENTS}

I would like to express my appreciation for all the support, productive feedback, and guidance provided by my dissertation chair, Jared Warren, Ph.D. I'd also like to extend a special thanks to my committee members, Gary Burlingame, Ph.D., Michael Lambert, Ph.D., Sally Barlow, Ph.D. and Joseph Olsen, Ph.D., for their thoughtful insights and careful reviews. Finally, I want to express a special thank-you to my husband, Chad, and my family for all their patience and moral support through this long and rigorous process. 


\section{TABLE OF CONTENTS}

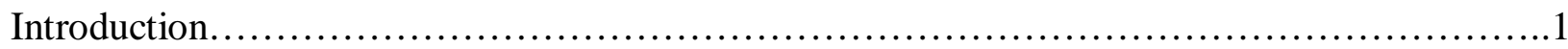

Child Psychotherapy Research................................................

Child/Adolescent Outcome Research in Usual Care.................................4

Mechanisms of Change in Child Psychotherapy....................................

Parent Functioning and Children's Mental Health..................................9

Parent Functioning and Child Psychotherapy Outcomes...............................15

Potential Impact of Child Treatment on Parent Symptoms...............................20

Limitations of Previous Research...........................................22

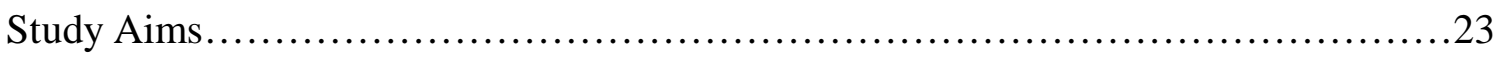

Hypotheses..................................................................24

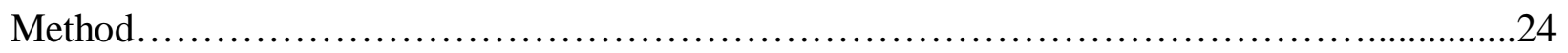

Participants............................................................... 25

Measures.................................................................26

Procedures...............................................................29

Analysis.....................................................................

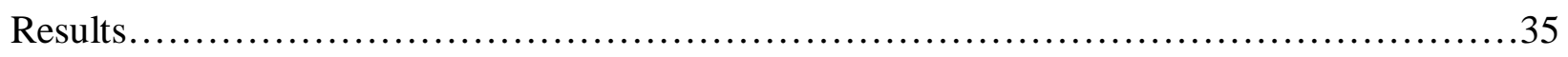

Hypothesis 1: Examining Whether Change Occurred in Parent Domains...............35

Hypothesis 2: Intake OQ Scores Predicting Changes in Y-OQ Scores and Intake

Y-OQ Scores Predicting Changes in OQ Scores.................................45

Hypothesis 3: Relationship Between Changes in OQ Scores and Changes in

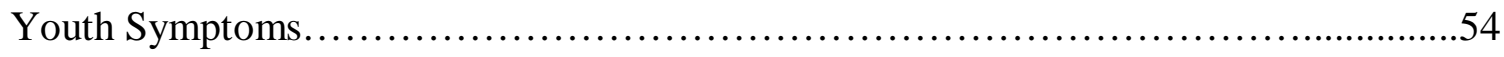

Discussion.......................................................................55 
Limitations.................................................................61

Study Applications and Future Directions.........................................64

References.....................................................................66 


\section{LIST OF TABLES}

Table 1 Intake Means and Standard Deviations of the OQ Domains and Y-OQ

Scores........................................................... 35

Table 2 Intraclass Correlation Coefficients for OQ Variables..........................36

Table 3 Fixed Effect Estimates, Random Variance, and Standard Errors for OQ Domain and OQ Total Change Trajectories........................................38

Table 4 Change Categories for OQ Scores.....................................43

Table 5 Change Categories for Clinical Sample OQ Scores.........................44

Table 6 Change Categories for Subclinical Sample OQ Scores........................45

Table 7 Estimates and Standard Errors for Intake OQ Scores Predicting Y-OQ Intake

Scores and Change....................................................47

Table 8 Estimates and Standard Errors for Intake OQ Scores Predicting Y-OQ_SR Intake

Scores and Change...................................................49

Table 9 Intake OQ Domains Predicting Premature Termination.......................51

Table 10 Estimates and Standard Errors for Intake Y-OQ Scores Predicting OQ Intake

Scores and Change.................................................52

Table 11 Estimates and Standard Errors for Intake Y-OQ-SR Scores Predicting OQ

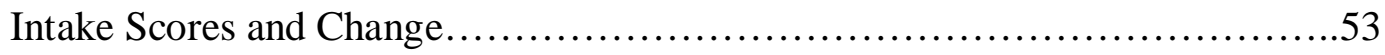

Table 12 Covariances and Standard Errors for OQ*Y-OQ Slope Interactions............54

Table 13 Covariances and Standard Errors for the OQ*Y-OQ-SR Slope Interactions....55 


\section{LIST OF FIGURES}

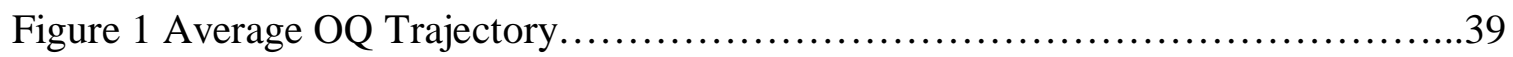

Figure 2 Average Symptom Distress Trajectory................................ 40

Figure 3 Average Interpersonal Relations Trajectory..............................41

Figure 4 Average Social Role Performance Trajectory.............................42

Figure 5 Social Role Performance at Intake Predicting Changes in Y-OQ Scores........48

Figure 6 Interpersonal Relations at Intake Predicting Changes in Y-OQ-SR Scores.....50 


\section{Parent Functioning and Child Psychotherapy Outcomes:}

\section{Predicting Outcomes in Usual Care}

Child and adolescent psychotherapy research has made considerable advances in recent decades. Over 1,500 treatment outcome studies have been conducted and many evidence-based treatments have been established for a broad range of problems and circumstances experienced by children and adolescents (Kazdin, 2003, 2004; Kazdin \& Kendall, 1998; Kazdin \& Nock, 2003; Kazdin \& Wassel, 2000). In spite of research supporting the efficacy of child psychotherapy, a number of important challenges and unanswered questions remain. One of the most notable deficiencies in child psychotherapy research has been the relative lack of studies examining treatment outcomes in "real-world” community settings (Garland, Hurlburt \& Hawley, 2006; Kazdin, 2003; Weisz, Jensen \& Mcleod., 2005; Weersing \& Weisz, 2002). Such extensions of research are important to lend evidence to theory, especially considering the important differences that exist between the way psychotherapy is conducted in controlled research settings and how psychotherapy is actually implemented and received in traditional community settings (Garland, Hurlburt \& Hawley, 2006; Kazdin, 2003; Weisz \& Kazdin, 2003; Weersing \& Weisz, 2002).

Another important deficiency in the literature has been the lack of studies examining mechanisms of change in child and adolescent psychotherapy (Kazdin, 2003, 2004, 2006; Weisz \& Kazdin, 2003; Weersing \& Weisz, 2002). This includes identifying moderators and mediators of successful therapeutic outcomes. The study of such mediators and moderators are particularly needed in traditional clinical settings because psychotherapy process research conducted in controlled clinical trials may not generalize appropriately to real-world contexts (Weisz et al., 2005). In addition, identifying mediators and moderators in traditional clinical settings has 
implications for the ways in which psychotherapy is implemented. For example, with a better understanding of underlying mechanisms of change in traditional practice settings, therapists may more effectively minimize the influence of factors known to negatively impact treatment and enhance the influence of factors known to improve treatment outcomes.

In examining mechanisms of change in child and adolescent psychotherapy, variables related to parent functioning may be among the most important factors to consider in predicting and promoting good child outcomes. Children’s dependence on their parents makes them particularly vulnerable to influences outside of their control including parent mental health, stress, marital and family functioning (Kazdin, 2003, 2004). Compelling evidence supports a significant relationship between parental distress and dysfunctional interpersonal relationships and the development of psychosocial problems in children (e.g. Beardslee, Versage, \& Gladstone, 1998; Crnic, Gaze \& Hoffman, 2005; Cummings \& Davies, 2002; Deater \& Decker, 1998). Further, emerging evidence indicates that parent distress and interpersonal relationships also impact psychotherapy outcomes for children (e.g. Kazdin, 2004; Kazdin \& Kendall, 1998; Kazdin \& Wassel, 2000; Reyno \& McGrath, 2006); however, this research is in its infancy (Kazdin, 2003). Consequently, the need remains for a clearer understanding of how child, parent, and family functioning or characteristics relate to child psychotherapy outcomes (Kazdin, 1994, 2003).

The purpose of the present study was to evaluate three important aspects of parent functioning — psychological symptom distress, interpersonal relations, and social role satisfaction — as potential predictors of successful treatment outcomes in a traditional community outpatient treatment setting for children and adolescents. Examining the associations between these variables is an important step toward identifying potential mechanisms of change in youth 
mental health treatment. More specifically, the study’s primary aims were threefold: 1) to examine whether parent distress, interpersonal relationships, and social role performance change over the course of the youth's treatment, 2) to examine how these factors as measured at intake predict youth progress in psychotherapy and 3) to examine whether changes in these parent factors over the course of youth psychotherapy are associated with changes in youth symptoms. Examining these issues provides valuable information on the importance of attending to parent distress and family relationships in both the assessment and treatment phase for children and adolescents receiving mental health services. In addition, this research may yield important implications for appropriately catering and adjusting psychological services to address parent symptoms and family relationships as well as child symptoms.

\section{Child Psychotherapy Research}

Effective psychotherapy for psychological and behavioral problems in children and adolescents is a critical need in the mental health profession. The lifetime prevalence rates for childhood psychopathology, although varied by disorder, age, sex, Socio Economic Status (SES), and ethnicity, are estimated to be around 14\%-22\% of all children and adolescents (Kazdin, 2003, 2004; Mash \& Dozois, 2003). This prevalence rate equates to approximately 14 million children and adolescents in the United States who experience significant impairment due to an emotional or behavioral problem (Kazdin, 2003). However, this prevalence rate does not capture the scope of impairment for affected children and adolescents, especially given the high comorbidity rates among diagnosed children and adolescents, which range from $50 \%$ to greater than 70\% (Kazdin, 2004). Beyond specific diagnosable emotional and behavioral problems, children and adolescents also frequently exhibit sub-threshold symptoms that also cause impairment, as well as “at-risk” behaviors such as alcohol abuse that may also warrant 
therapeutic attention (Kazdin, 2003, 2004). Furthermore, child psychopathology has significant lifelong consequences for both the child and society, as many adult problems have their origin in childhood and represent a continuity of dysfunction across the lifespan (Kazdin, 1991: Mash \& Dozois, 2003). Consequently, early and effective interventions are needed to provide relief to children and adolescents and to decrease the risk of continued and exacerbated impairment across the lifespan.

Research on child and adolescent psychotherapy has made great advances in recent decades (Kazdin, 2003), with over 1,500 treatment outcome studies conducted (Kazdin, 2003; Kazdin \& Kendall, 1998; Kazdin \& Wassel, 2000). Interventions and techniques have grown considerably, with over 550 named psychotherapies in use (Kazdin, 2006). Many evidence-based treatments have been established for various child clinical disorders including anxiety, depression, oppositional and conduct disorder and ADHD (Kazdin \& Nock, 2003).

This growing literature provides strong evidence that psychotherapy works for children and adolescents with efficacy studies revealing large effect sizes compared to no treatment (Kazdin, 2003; Weisz \& Kazdin, 2003). In a review of meta-analyses Weisz and colleagues (2005) reported that the effect size difference between children in therapy compared to control groups was medium to large (effect size for each broad based meta-analysis reviewed ranged from .71 to .84) indicating consistent beneficial effects of treatment. Thus, youth who participate in therapy are better off than those who do not receive treatment (Kazdin, 2003; Kazdin \& Nock 2003; Weisz et al. 2005; Weisz \& Kazdin, 2003).

\section{Child/Adolescent Outcome Research in Usual Care}

In spite of research supporting the efficacy of child psychotherapy, a number of important challenges and unanswered questions remain. Although research demonstrates the efficacy of 
many evidence-based treatments for children and adolescents, an important concern is the extent to which such findings may generalize to traditional clinical practice (or "usual care”) settings (Garland et al., 2005; Kazdin, 2004; Weersing \& Weisz, 2002; Weisz \& Kazdin, 2003). Relatively few studies have examined the effectiveness of psychotherapy for children and adolescents in traditional clinical settings (Garland et al., 2005; Weersing \& Weisz, 2002). Of the studies executed in traditional community settings, some provide supporting evidence for its effectiveness, but most reports are discouraging with treatment effects near zero (Garland et al., 2005). Further, premature drop-out rates are high in traditional community settings, with estimates between $28 \%$ and $85 \%$ of all children who begin treatment (Garcia \& Weisz, 2002; Kazdin, 2003; Kazdin \& Wassel, 1998; Pina, Silverman, Weems, Kurtines, \& Goldman, 2003). This question of generalizability to clinical settings along with the sobering realization of attrition, paucity of research, and some discouraging initial findings in traditional practice underscore the urgent need for increased attention to treatment processes and outcomes in community settings (Kazdin, 2004).

The question of generalizability to community settings is particularly relevant given the significant gap between how psychotherapy is studied in laboratory settings and how treatment is most often implemented in regular clinical practice (Kazdin, 2003, 2004; Weisz \& Kazdin, 2003). Clinicians practicing in traditional community clinics are often skeptical about the utility of research findings from controlled clinical trials due to the disparity of conditions between highly controlled studies and actual clinical practice (Garland et al., 2005; Weisz \& Kazdin, 2003). Most of the available research on child and adolescent psychotherapy outcomes is conducted in controlled laboratory settings where participants are recruited (Kazdin, 2003; Weersing \& Weisz, 2002). There are many individual, family, and treatment factors that differ 
between recruited participants and referred participants, such as those found in traditional clinical settings. For example, recruited children and adolescents who participate in clinical trials often demonstrate less severe symptomatology (Kazdin, 2003, 2004) and are often screened to avoid comorbid diagnoses (Weersing \& Weisz, 2002). On the other hand, clients in traditional clinical settings often present with comorbid diagnoses and more severe symptoms and disturbance (Kazdin, 2003, 2004; Weisz \& Kazdin, 2003; Weersing \& Weisz, 2002). Family characteristics also frequently differ between recruited participants and referred participants where parents of referred participants exhibit greater psychiatric dysfunction and stress, more impairment and socioeconomic disadvantage, and less motivation for treatment (Kazdin, 2003, 2004; Weisz \& Kazdin, 2003). Further, treatment itself is almost always qualitatively and quantitatively different from a laboratory setting to a traditional clinical setting (Kazdin, 2003). In most controlled child and adolescent treatment outcome studies, treatment is fixed in duration, uses treatment manuals, is carefully monitored for adherence to the treatment protocol, and is typically delivered by closely supervised doctoral students in clinical psychology. In traditional clinical practice, treatment is often varied in duration, makes use of various methods and interventions, is typically not concerned with following a specific treatment protocol nor monitoring protocol adherence for outcome, and is provided by clinicians from varied academic and professional backgrounds (Kazdin, 2003, 2004; Weisz \& Kazdin, 2003). Further, most community service providers identify themselves as eclectic in regards to theoretical orientation (Garland et al. 2005) as opposed to controlled clinical trials which mostly adhere to a specific type of treatment. These differences limit the external validity of controlled research trials and call into question their generalizability to real world settings. 
In addition, more research is needed to examine how therapy and processes in traditional community settings impact child, adolescent, and family outcomes (Garland et al., 2005). Weisz and Kazdin (2003) assert that examining real-world factors (often viewed as nuisances to be avoided in controlled clinical trials) is exactly what is needed to best understand the workings and implementation of psychosocial treatment in traditional community settings. Thus, extending treatment research to typical clinical settings is critical to establish true effectiveness of therapy, build robust and practical treatments, and lend evidence to theory.

\section{Mechanisms of Change in Child Psychotherapy}

In addition to the need for increased emphasis on treatment outcomes in traditional community settings, one of the most frequently cited issues in the child treatment literature is the need for increased attention to understanding predictors of positive treatment outcomes and the underlying mechanisms of change in therapy (e.g. Kazdin, 2004; Weisz \& Kazdin, 2003; Weersing \& Weisz, 2002). Weersing and Weisz (2002) note that when psychotherapy is effective for youth, the underlying mechanisms of change-how therapy works — are not well understood. Kazdin and Kendall (1998) call for a more comprehensive picture of effective treatment: what it constitutes, how and why it works, and for whom it is most effective. Kazdin (2006) argues that the identification of the processes and mechanisms through which therapy works on the individual are the most pivotal need in psychotherapy research for children and adolescents. Knowledge of factors that influence treatment outcome can aid in theory development regarding the putative causes and mechanisms of change in therapy (Kazdin, 2006; Weisz \& Kazdin, 2003).

A better understanding of the change mechanisms in youth psychotherapy may help to tailor interventions to minimize the influence of factors known to negatively impact treatment 
and enhance the influence of factors known to improve treatment outcomes. According to Kazdin (2006), "If we wish to optimize therapeutic change, understanding the critical ingredients and processes through which they operate is essential” (p. 82). Using those critical ingredients, we may refine treatment procedures to enhance their effectiveness for children at high risk for treatment failure (Berman, Weems, Silverman, \& Kurtines; 2000) and directly target those factors that contribute to or maintain adjustment problems in the child.

Currently, there is a particular need for studies of change processes in usual care settings. Weisz and colleagues (2005) note that the efficacy of evidence-based treatments for children and adolescents is well established due to clinical research trials, but there is a significant lack of knowledge regarding the "effectiveness" of psychotherapy because mechanisms of change have not been widely examined in "ordinary” clinical conditions. This is particularly relevant given differences between controlled clinical trials and traditional clinical practice. Thus, identifying effective and ineffective elements of usual practice will improve evidence-based practice and help close the gap between research and practice (Garland et al., 2005). These real-world tests are vital; for example Weisz and colleagues (2005) asserted:

the most valid answers to questions about treatment outcome, component contributions, moderators, and mediators are less likely to come from research with recruited samples seen under controlled conditions than from referred samples seen under representative clinical practice conditions. (p. 31)

Consequently, a better understanding of change processes in real world clinical settings are of great importance in promoting therapeutic change for the millions of youth served in these settings (Kazdin, 2003, 2006; Weisz et al., 2005). 


\section{Parent Functioning and Children's Mental Health}

There are numerous potential mediators and moderators of treatment outcome for children and adolescents. Of the many potentially significant variables, parent characteristics may be among the most important to consider given the substantial influence parents have on their children (Kazdin, 1994, 2003). The dependence of children on their parents makes them particularly vulnerable to influences outside of their control including parent mental health, stress, marital and family functioning, and socioeconomic status (Kazdin, 2003). Parent influence on children’s growth and development has long been recognized (e.g. Kazdin, 2004). Compelling evidence supports a significant relationship between parent factors, including psychopathology, stress and interpersonal relationships, and the development and maintenance of child psychopathology (e.g. Anselmi, Piccini, Barros, \& Lopes, 2004; Downey \& Coyne, 1990; Hammen, Brennan \& Shih, 2004; Jaser, Langrock, Keller, Merchant, Benson, Reeslund, Champion \& Compas, 2005; Reyno \& McGrath, 2006). These parent factors are particularly important given that children are rarely self-referred for treatment. That is, unlike adult clients, they are most often brought to treatment because parents have concerns about their behavior and emotional functioning. Consequently, when premature termination of treatment occurs, most often, parents or guardians make this decision (Garcia \& Weisz, 2002). Unfortunately, the processes and characteristics of parents and families that affect treatment have been largely neglected in the child psychotherapy research to date (e.g. Cummings \& Davies, 2002; Kazdin, 2004). As a result, examining the ways in which parent and family characteristics relate to child and adolescent psychotherapy outcomes is an important need in the psychotherapy literature (Kazdin, 1994, 2003). 
Parent and child psychopathology. Compelling evidence supports the assertion that parental psychopathology influences children’s behavior and adjustment. For example, Anselmi and colleagues (2004) found that of the factors they examined, maternal psychiatric disorder was most correlated with and explained the most variance in child behavioral problems. Of the studied parental psychopathologies, the bulk of studies have examined the significant relationship between parent depression and child affective disorders (e.g. Beardslee et al., 1998; Downey \& Coyne, 1990; Hammen et al., 2004; Jaser et al., 2005), with evidence that children of depressed parents meet criteria for major depression at a rate of six times higher than control children (Downey \& Coyne, 1990). Similarly, these children are also at greater risk for experiencing general difficulties in functioning, interpersonal relations, and attachment (Beardslee et al., 1998). Furthermore, significant negative long term effects from living with a depressed parent are noted in the literature (e.g. Beardslee et al. 1998; Hammen, 1990).

Beyond parental depression, other parent psychopathologies and symptoms have been investigated as they relate to child psychopathology. Some of these parent variables include antisocial behaviors and personality, substance abuse and dependency, and anxiety. For example, in a study investigating parent variables in relation to conduct disorder in children, McBurnett, Pfiffner, Capasso, Lahey and Loeber (1997) found that parent antisocial behaviors and personality along with substance abuse and dependency were positively related to nonaggressive conduct disorder symptoms in their children, while poor parent-child relations were associated with a high level of aggressive and non-aggressive conduct behaviors. In regard to parental anxiety disorders, Cobham, Dadds and Spence (1998) noted that parents of anxious children were more likely to meet criteria for an anxiety disorder than parents of nonanxious 
children. These findings, among others, strongly suggest that parent psychopathology influences the development of child psychopathology.

The exact pathways through which parent psychopathology influences child psychopathology are difficult to distinguish. For example, it is frequently recognized that many psychopathologies, including depression, share a significant genetic component, which alone increases children's risk for psychopathology, although this relationship is often ignored in the literature. Parental psychopathology also influences the environment in which the child resides and grows and changes the ways in which parents interact with their children. For example, in extreme cases parental psychopathology may be related to increased child maltreatment (e.g. Walker, Downey, \& Bergman, 1989), which directly negatively impacts youth adjustment. However, parental psychopathology is more frequently associated with negative parenting practices. For example, maternal depression—-the most frequently studied and noted parent psychopathology in the literature-involves negative parenting practices and parent-child relationships. Specifically, depressed parents often display negative parenting behaviors such as withdrawal, unresponsiveness, little positive affect, slower response, or intrusive, irritable, and hostile behavior toward their children (Downey \& Coyne, 1990; Jaser et al. 2005). Parental depression is also associated with poor child affective interaction and an insecure attachment relationship (Anselmi et al., 2004). Such negative parenting behaviors in depressed parents are correlated with increased internalizing and externalizing problems in their children (Marchand \& Hock, 1998). Thus, negative parenting practices that result from parental depression appear to play a role in the development and maintenance of child and adolescent maladjustment (e.g. Gallimore \& Kurdek, 1992). 
The complex relation between parent and child psychopathology is further emphasized by the recognition that the relation between parent and child symptoms and functioning is not uni-directional. It is understood that children may influence parent functioning in addition to parents influencing child functioning (e.g. Deater-Deckard, 1998; Kazdin \& Wassel, 2000; Papp, Cummings \& Goeke-Morey, 2005). For example, a child exhibiting symptoms of psychopathology may be difficult to parent, which subsequently increases parental stress and parent-child discord and exacerbates parent symptoms of psychopathology. This may perpetuate a downward spiral of continued distress, dysfunctional family interactions and parenting behaviors (e.g. Anselmi et al., 2004; Downey \& Coyne, 1990; Jaser et al. 2005), leading to exacerbated child symptoms (e.g. Gallimore and Kurdek, 1992; Marchand \& Hock, 1998).

In summary, there is considerable evidence to support the relationship between parental psychopathology and child psychopathology. However, the relationship is complex involving other potential moderators and partial mediators such as shared genes, parenting practices, and reciprocal child influences on parent symptoms. Although these factors make it difficult to draw definitive conclusions regarding the relationship between parent and child symptoms, the current literature provides a strong foundation for the notion that parent psychopathology plays a substantial role in children's mental health.

Family interpersonal relationships and child functioning. Similar to the relationship observed between parent psychopathology and child adjustment, family interpersonal relationships play a key role in child functioning. For example, marital discord and parent-child conflicts are frequently cited as significant contributors to youth maladjustment. Further, disruptions in one relationship within the family rarely occur without negatively influencing other relationships (Cox, Blair, \& Harter, 2001; Erel \& Burman, 1995; Harold et al., 2004). 
Consequently, it is beneficial to consider the ways in which both the interparental relationship and the parent-child relationship influence child psychosocial adjustment.

Marital discord is often related to child psychopathology, including internalizing and externalizing disorders (Cummings \& Davies, 2002; Grych \& Fincham, 1990; Harold, Shelton, Goeke-Morey \& Cummings, 2004; Zimet \& Jacob, 2001). Upon exposure to interparental conflict, children experience manifold emotional reactions, including fear and anger (Cummings \& Davies, 2002). Cummings and Davies (2002) suggest that internalizing disorders results directly from exposure to marital conflict, while externalizing disorders result from changes in parenting behaviors as a consequence of marital discord. In a review of the literature on marital conflict as influential of child adjustment, Grych and Fincham (1990) found that frequent exposure to interparental conflict increases distress and maladaptive behavior in children, with more severe conflict associated with higher child distress. Further, children of all ages are negatively affected by interparental conflict, evidenced across a variety of types of interparental conflicts including nonverbal expressions of anger and marital withdrawal to physical violence (Cummings \& Davies, 2002; Grych \& Fincham, 1990). In regard to older youth, Harold and Conger (1997) found that adolescent report of interparental hostility significantly predicted subsequent externalizing and internalizing symptoms in their sample of 370 seventh-graders. Finally, the observed relationship between child behavior problems and marital conflict is stronger in clinical samples compared to non-clinical samples (Grych \& Fincham, 1990).

There are numerous ways in which marital conflict indirectly and directly influences child and adolescent psychosocial adjustment. Most frequently noted in the literature are the mechanisms of modeling, emotional insecurity, the spillover effect, and cognitive contextual influences (Zimet \& Jacob, 2001). For example, Harold and colleagues (2004) reported in their 
study examining marital conflict, emotional security, and children's psychological adjustment that marital conflict negatively influenced children's psychological adjustment through influencing the child's feelings about the security of their relationship with their parents. In a meta-analysis of 68 studies, Erel and Burman (1995) found that marital conflict influences the parent-child relationship quality through the spillover hypothesis, which is the expression of feelings within the marital relationship produce the same feelings within the parent-child dyad. Cognitive-contextual influences acknowledge that children's appraisal of threat and interpretation and self-blame may mediate interparental conflict and child adjustment (Zimet \& Jacob, 2001). Further, as mentioned earlier, interparental conflict likely compromises parenting behaviors and the parent-child relationship which negatively influences child psychosocial adjustment (Cummings \& Davies, 2002). More in-depth explanations for the observed relationship between marital conflict and youth adjustment have been offered and are described elsewhere (e.g. Cummings \& Davies, 2002; Grych \& Fincham, 1990; Harold \& Conger, 1997; Zimet \& Jacob, 2001).

Although marital discord negatively impacts the parent-child relationship and subsequent child behavior, the parent-child relationship is uniquely influential on child psychopathology. This relationship is frequently observed through parenting practices as described previously (e.g. Anselmi et al., 2004; Deater-Decker, 1998; Downey \& Coyne, 1990; Jaser et al. 2005). The parent-child relationship is also significantly related to the dimensions of parental psychopathology and parenting stress. Due to compromised and negative parenting practices associated with parental psychopathology, the parent-child relationship is at greater risk to be compromised in the presence of parental psychopathology (Papp, et al., 2005). Using structural equation modeling, Papp and colleagues found in their study of 277 families involved in a 
broader investigation of family processes, that the parent-child relationship accounted for a significant portion of variance between parent psychological distress and child adjustment problems (2005). Specifically, parenting qualities mediated the relationship between parent psychological distress and child maladjustment (Papp et al., 2005). Taken together these studies provide evidence that family interpersonal relationships influence the development and maintenance of child psychopathology.

Finally, many of the studies examined above illustrate the important observation that parent psychopathology, stress, and dysfunctional interpersonal relationships and interactions frequently co-occur. Parents who experience psychopathology also frequently manifest negative and maladaptive parenting practices and have negative relationships within their families, which serve to impact child adjustment and psychopathology. For instance, Reyno and McGrath note that maternal depression is correlated with higher stress and discord as well as other parent psychopathologies and lower SES (2006). Further, Deater-Decker (1998) notes that high parenting stress has been linked to child depression, separation anxiety, and drug use. Specifically, parenting stress directly influences parenting behavior, leading to more authoritarian parenting styles and less parental responsiveness. This, in turn, directly influences child behavior, leading to poorer cognitive, social, and emotional development (Deater-Decker, 1998). These reviews suggest that these factors frequently co-occur and all influence child psychopathology. Through their influence, these factors may explain, at least in part, the development and maintenance of both internalizing and externalizing childhood disorders. Parent Functioning and Child Psychotherapy Outcomes

In addition to its relation to children's general behavioral and emotional functioning, parent functioning also appears to play an important role in children’s response to 
psychotherapy. Research on the predictors of treatment outcome for children and adolescents frequently cites parental psychopathology and family interpersonal relationships as salient factors (Kazdin, 1995, 2003, 2004; Kazdin \& Kendall, 1998; Reyno \& McGrath, 2006). This holds true across a variety of problems for which children and adolescents are referred for treatment.

Successful treatment for child and adolescent externalizing disorders appears to be significantly related to parental distress and psychopathology. This finding is especially noteworthy as externalizing disorders are well represented in both therapy research and clinical practice, being the most frequent category of referrals for treatment in children (Kazdin, 2003). In a meta-analytic review of the barriers to treatment with parent training for children with externalizing disorders, Reyno and McGrath (2006) found numerous parent factors that negatively influenced treatment outcome including parenting stress, negative life events, and maternal depression. Specifically, maternal depression was frequently and moderately related to poorer treatment outcomes (Reyno \& McGrath, 2006). Further, better treatment outcomes were found when parent stress and psychopathology were specifically addressed in treatment (Reyno \& McGrath, 2006). Comparable findings were observed by Webster-Stratton and Hammond (1990) in their study of a parent training program for young children (ages 3 to 8) with conduct problems in which parental depression was predictive of children's conduct disorder symptoms at the end of treatment.

Kazdin, Holland and Crowley (1997) also examined how parent and child factors negatively influenced treatment participation for children and adolescents experiencing externalizing disorders. Specifically, in their study of 242 children and adolescents receiving services for externalizing disorders, parent and family stressors and parent history of antisocial 
behaviors were among the most significant factors in predicting premature treatment dropout. Similarly, Kazdin and Wassel (1998) studied several domains that they predicted would influence treatment completion and outcome in 304 children with externalizing disorders in an outpatient setting. Although parents of children who dropped out of treatment did not show greater psychopathology and stress, parental psychopathology and stress were related to their children's improvement in treatment for those who remained in treatment. That is, children who improved had parents who showed less dysfunction (Kazdin \& Wassel, 1998).

Beyond being related to psychotherapy outcomes for externalizing disorders, parent distress also appears to be related to treatment outcomes for children and adolescents with internalizing disorders. For example, Brent et al. (1998) found that treatments for internalizing disorders are less effective when applied to children of depressed mothers. Specifically, they found that cognitive behavioral therapy (CBT) was not as effective in the presence of maternal depressive symptoms. Similarly, in their treatment of child anxiety disorders, Berman and colleagues (2000) found that predictors of treatment outcome using exposure-based CBT methods included parental psychopathology symptoms, especially depression as measured by the BDI. More specifically, they found that parental global severity of symptoms, as measured by the Symptom Check-List (SCL-90), were significantly higher for the treatment failure group compared to those who succeeded in treatment. They also found that parental symptoms were important predictors of reduced symptom severity in children and teens. Notably, as the age of the client increased the importance of parental psychological symptoms in predicting outcome diminished. The authors concluded that parental psychopathology is thus more problematic when treating younger children compared to adolescents (Berman et al., 2000). Comparable findings were observed by Cobham and colleagues (1998) in their study of CBT treatment for child 
anxiety disorders with 67 children between the ages of 7 and 14 diagnosed with an anxiety disorder. The authors found that anxious children who also had an anxious parent responded less favorably to CBT treatment for anxiety than anxious children whose parents were not anxious. Further, if parent anxiety was targeted in therapy along with providing CBT for the child, outcomes in children with anxious parents were improved (Cobham, Dadds, \& Spence, 1998).

Finally, interparental and parent-child relationship factors appear to be important predictors of youth psychotherapy outcomes, and are frequently studied in conjunction with parent psychopathology and parenting stress. Just as marital discord is observed to influence child adjustment and psychopathology, marital discord is also influential for child psychotherapy outcomes. Although more research is needed to clarify the magnitude of the relationship between marital discord and child psychotherapy outcomes, previous research has estimated that this relationship accounts for approximately $10 \%$ of explained variance in child psychotherapy outcome (Zimet \& Jacob, 2001). However, this finding is based on many studies that utilized nonclincal samples with lower levels of family conflict and psychopathology (Zimet \& Jacob, 2001); greater effect sizes may be evident in clinical populations.

Cummings and Davies (2002) emphasize the need for research on how to effectively intervene within dysfunctional marital relationships to improve the well-being and adjustment of children. Specifically, they call for "systematically tracing the bidirectional and transactional interplay between child, parent, parent-child, and interparental functioning” (p. 46) as a key focus for understanding child adjustment. Thus, interparental relationships and conflict influence their children’s progress and outcome in psychotherapy. However, the parents’ marital relationship is rarely a target of assessment or intervention in youth psychotherapy (Kazdin, 2004). 
The parent-child relationship is also an influential factor in child psychotherapy outcomes (Kazdin, 2004). For instance, parent management training is a frequent method of psychotherapy for children and teens and is based on the assumption that maladaptive reactions in children are inadvertently developed and sustained through dysfunctional parent-child interactions (Kazdin, 2004). Thus, the ways in which parents interact with their children, as well as the quality of their interpersonal relationships impact treatment attendance and progress.

Calam and colleagues (2002) examined how the mother-child relationship, maternal depression, and parenting stress were related to therapy attendance for children referred for behavior problems. They found significant differences between mothers who brought their children to therapy versus those who did not. Specifically, they observed that mothers who were non-clinic attendees were more critical and hostile towards their children, and evidenced more depression and more parenting stress (Calam, Bolton \& Roberts, 2002). Despite their limited sample size of 57 mothers, these findings emphasize the need to assess and attend to parent-child interactions as they relate to therapy attendance and drop-out.

Kazdin (1995) evaluated how family dysfunction, along with other parent and child variables, related to treatment outcome in conduct disordered youth. Kazdin found a significant association between treatment responders versus non responders, in that family relations, parent stress, parent psychopathology and child severity were related to treatment responsiveness. Specifically, family dysfunction, parental stress and psychopathology were related to level of severity of presenting child conduct symptoms at intake of treatment and prosocial functioning at end of treatment, thus providing support that these factors influence treatment outcome. From this study, Kazdin calls for more research examining the ways in which family dysfunction and parent variables moderate or mediate child psychotherapy outcome (1995). 
Similarly, in a study of family characteristics of 106 referred children with externalizing disorders, Hemphill and Littlefield (2006) found that over the course of treatment, positive parent-child interaction was one of two main predictors of reductions in externalizing and internalizing behaviors. The authors suggest from these findings that successful treatment outcomes are more likely for children whose parents who show warmth and affection. However, the exact mechanisms of change in this study were unclear, warranting further attention to how parent-child interactions influence psychotherapy outcomes for children (Hemphill \& Littlefield, 2006). In summary, ample research supports the notion that parent psychopathology and parents’ interpersonal relationships play an important role in child treatment outcomes.

\section{Potential Impact of Child Treatment on Parent Symptoms}

Not surprisingly, child symptom change has been the primary outcome of interest in child psychotherapy research (Kazdin, 2004). Evaluating change in child symptoms is an essential feature of child psychotherapy research; however, treatment may have broader effects that extend to changes in the parent or family (Kazdin, 2004, 2006; Kazdin \& Wassel, 2000). As previously noted, the relationship between parent functioning is bi-directional. Although rarely the focus of study in the treatment outcome literature, addressing child symptoms may in turn influence parent and family factors (Kazdin, 2004; Kazdin \& Wassel, 2000). Kazdin and Wassel (2000) assert that improvements in parent and family functioning might be one of these broader treatment effects; however, this has not been widely studied. In fact, the study by Kazdin and Wassel (2000) appears to be the first of its kind in examining specifically how parent symptoms and family functioning change over the course of child psychotherapy.

Kazdin and Wassel (2000) examined how child, parent, and family functioning changed over the course of a specific evidence-based treatment for 250 children referred for oppositional, 
aggressive, and antisocial behavior. Although all parents received parent management training, the authors reported that neither parent characteristics, parent functioning, nor family functioning were topics of direct focus in treatment (Kazdin \& Wassel, 2000). As hypothesized, child, parent, and family symptoms improved over the course of treatment with a large effect size for children and a small to medium effect size for parents. The authors found that reductions in child symptoms significantly correlated with reductions in parent symptoms and improvement in family functioning. The authors concluded that the improvements in symptoms across children, parents, and family are moderately related over the course of treatment. This suggests that child treatment for conduct problems has broader effects on improving parent symptoms and family functioning.

This study is unique and important as most child treatment outcomes studies restrict their evaluation of treatment effectiveness to changes in child symptoms only (Kazdin, 2004; Kazdin \& Wassel, 2000). This habit neglects potential clinically relevant areas that are also influenced by therapy such as parent symptoms and family functioning (Kazdin, 2004). These factors are important as they may serve to continue to influence child symptoms, as discussed previously, as well as serve to maintain therapeutic changes (Kazdin, 2004; Kazdin \& Wassel, 2000). Further, such symptoms are clinically relevant for parents as parental psychopathology and dysfunctional and dissatisfying interpersonal relationships may be indirectly targeted and alleviated in this manner. This evidence has implications for conceptualizing how therapy works as well as how it may be catered and delivered to children and adolescents. "Quite possibly, the scope of the benefits of child treatment is being overlooked and underestimated" (Kazdin \& Wassel, 2000, p. 219). The authors conclude that more research is needed to examine mechanisms in child 
psychotherapy that influence child and parent outcomes as well as the timing of those changes throughout treatment (Kazdin \& Wassel, 2000).

\section{Limitations of Previous Research}

As noted previously, one of the most frequently cited limitations in the child psychotherapy literature is the need for increased attention to potential mechanisms of change. An important next step in this area of research is to identify variables that are associated with change in youth outcomes. Relatively few studies have examined how change in other variables are associated with change in youth symptoms. Identifying such variables is essential to understanding the underlying mechanisms at work in child and adolescent psychotherapy and provides a foundation for more formal tests of mediation among these variables.

Further, the need to identify mechanisms of change is particularly important in traditional community settings. Research that identifies mechanisms of change in child and adolescent therapy is rarely conducted in traditional community settings. This gap is significant given the salient differences between usual care settings and controlled clinical laboratory research trials. Identifying mechanisms of change in these usual care settings will lend evidence to theory born out of controlled clinical trials and increase the utility and applicability of these findings.

Finally, the literature provides compelling evidence for the connection between parent functioning and the development and maintenance of child psychopathology as well as influencing child psychotherapy outcomes. However, the specific aspects or domains of parent functioning that influence child psychotherapy outcomes are not well understood. Furthermore, research examining the influence of parent functioning on child psychotherapy outcomes is particularly sparse in usual care settings. 
An important contribution to the child psychotherapy literature will be to examine the potential impact youth psychotherapy may have on parent functioning. It stands to reason that as youth symptoms improve, parents may report decreased symptomatology and increased satisfaction with their interpersonal relationships and social roles. The examination of these domains as they relate to youth psychotherapy outcomes has been neglected but is relevant to how parents may benefit from their children receiving services. Further, all these domains may be differentially related to youth treatment outcome, but this has never been examined.

\section{Study Aims}

The purpose of the present study was to examine parent characteristics that may influence the relationship between child psychotherapy outcomes in real world, clinical settings. More specifically, the study's primary aims were threefold: 1) to examine whether parent distress, interpersonal relationships, and social role performance change over the course of the youth's treatment, 2) to examine how these factors as measured at intake predict youth progress in psychotherapy and 3) to examine whether changes in these parent factors over the course of youth psychotherapy are associated with changes in youth symptoms.

This study holds significant clinical importance and benefits to child and adolescent psychotherapy. Specifically, identifying predictors of treatment success or failure-such as parent symptomatology and interpersonal relationships—-helps clinicians be aware of clients that may drop out of treatment or be less likely to respond. In addition, findings underscore the importance of attending to these parent factors both in the assessment and treatment phases for children and adolescents presenting for mental health services. Finally, this study also demonstrates the ways in which parents indirectly benefit from their children receiving 
psychological services, expanding our current limited view of derived therapeutic benefits in child psychotherapy.

Hypotheses

Based on the literature reviewed above, I hypothesized:

1. The three domains of parent functioning—symptom distress, interpersonal relations and social roles—-would improve over the course of child/adolescent treatment.

2a. Parent symptom distress, interpersonal relations and social role performance at intake would be significant predictors of youth scores at intake, youth rate of change over the course of treatment, and premature termination.

2b. Secondly, youth symptoms at intake would significantly predict parent symptom distress, interpersonal relations, and social role performance at intake and parent rate of change over the course of treatment.

3. There would be a significant relationship between the progression of symptom changes in both children and adolescents and their parents' domains of symptom distress, interpersonal relations and social roles.

\section{Method}

This project was part of a larger research study examining child and adolescent treatment outcomes in a community mental health system. 


\section{Participants}

Children aged 4 to 17 years and their primary caregivers were recruited for participation through the Valley Mental Health Child Outpatient Clinic in Salt Lake City, Utah. Participants received outpatient treatment through a large public community mental health system that serves an area of over 1 million residents. At their initial intake appointment, potential participants were informed of the study and asked to participate in research to learn more about the things that may predict or enhance positive treatment outcomes in child and youth receiving psychological services. Participation was completely voluntary.

The initial sample included 350 children and adolescents; however, 11 were excluded because they were medication management cases without psychotherapy. Of the 339, 140 were females (41.3\%) and 189 were males (55.8\%). Participants came from diverse backgrounds based on parent identification of ethnicity. Forty-three (12.3\%) of the children were Mexican American, 12 (3.4\%) were Hispanic (not from Mexico), 18 (5.3\%) were African American, 245 (72.3\%) were Caucasian, and 66 (19.5\%) indicated that they were of other groups or mixed background. As expected in a traditional community setting, children presented with varying symptoms and diagnoses. The most common primary diagnoses were ADHD ( $n=97 ; 27.7 \%)$, adjustment disorder ( $n=56 ; 16 \%)$, depression ( $n=54 ; 15.4 \%)$, oppositional defiant disorder $(n=27 ; 7.7 \%)$ and anxiety disorders $(n=23,6.6 \%)$. The remainder had a variety of other primary diagnoses. As might also be expected from a community sample, there was a high rate of comorbidity with $65 \%$ ( $n=227)$ of the participants having multiple diagnoses. These diagnoses were obtained from the interviewing clinician at intake.

To more closely examine the question of generalizability of study results to the greater population being served at the clinic used in this study, chi-square analyses were run using 
available demographic information for all clientele presenting at the clinic during the same time period of this study's data collection. Specifically, chi-square analyses were run using study participants and archival clinic demographic data on ethnicity and gender. Results from these analyses indicated non-significance between the proportion of total minority clients presenting at the clinic and the proportion of minority clients participating in this study. Similar nonsignificant results were found for the proportion of male to female clientele presenting at the clinic and the proportion of male to female clients participating in this study This suggests that in relation to these specific demographic parameters, the sample of this study is representative of the typical clientele for this clinic. In regards to symptom severity of those who participated in this study compared to non-participants, a prior study indicated that the average intake Y-OQ score for a sample of 1,782 clients presenting at the clinic between the years of 1997 and 2008 was 82.2 (Warren, Nelson \& Burlingame, in press). This score is lower than the average intake Y-OQ score in this present study of 87.8, indicating that youth participating in this study displayed slightly higher psychosocial distress at intake.

\section{Measures}

Parent functioning. The Outcome Questionnaire-45 (OQ-45; Lambert et al., 2004) was used as a measure of psychological distress for parents. The OQ-45 is a 45 -item self-report measure of adult symptomatology designed to be administered on a repeated measures basis and sensitive to change. The OQ-45 is scored using a 5-point scale (0 - never, 1 - rarely, 2 sometimes, 3 - frequently, 4 - almost always). The OQ-45 yields a total score as well as three subscale scores. The total score ranges from 0 to 180 with higher scores indicating greater pathology. A cutoff score of 64 or greater indicates significant dysfunction and a change in 14 points over time evidences reliable change (Lambert et al., 2004). 
The three subscales of the OQ-45 measure three domains of functioning typically endorsed by psychologically distressed persons, including 1) Symptom Distress 2) Interpersonal Relations, and 3) Social Role performance. The Symptom Distress subscale (SD) is composed of questions assessing symptoms corresponding to the most frequently presented mental disorders with numerous items specifically addressing anxiety and depression. A score for this subscale ranges from 0-100 with a cutoff score of 36/37 and is obtained by summing the client's ratings on the corresponding 25 items. Reliable change on this scale is evidenced in a decrease in 10 or more points. The Interpersonal Relations subscale (IR) assesses satisfaction and difficulties in interpersonal relationships with friends and family. Research indicates that good interpersonal relationships are critical for psychological well-being and individuals who attend therapy frequently evidence interpersonal relational difficulties as evidenced by the frequency with which such difficulties are addressed in psychotherapy (Lambert et al., 2004). The Interpersonal Relations score ranges from 0-44 with a cutoff score of $15 / 16$ and is obtained by summing the corresponding 11 items. Reliable change on this subscale is indicated if the individual's initial score changes 8 or more points. The Social Role subscale (SR) examines client's levels of distress, dissatisfaction and inadequacy in relation to vocational, recreational and family roles. The score or the Social Role domain ranges from 0-36 with a cutoff score of 12/13 and is obtained by summing ratings on the corresponding 9 items. Reliable change is evidenced in this scale with a decrease of 7 or more points (Lambert et al., 2004).

The OQ-45 is a well-established instrument that has been used both nationally and internationally with a diverse range of populations. The overall OQ-45 has a reported internal consistency of .93 and a three-week test-retest reliability of .84, well within adequate ranges (Lambert et al., 2004). The three subscales also demonstrate adequate reliability and internal 
consistency. The Symptom Distress subscale has a reported internal consistency of .91 and testretest Reliability of .78. The Interpersonal Relations subscale has an internal consistency of .74 and test-retest reliability of .80. Finally, the Social Role subscale has an internal consistency of .71 and a test-retest reliability of .82. The OQ-45 also demonstrates adequate concurrent validity with numerous related measures. Confirmatory factor analysis appears to support an overall unidimensionality of the OQ-45, as well as an underlying three-factor model (Mueller, Lambert \& Burlingame, 1998; Lambert et al., 2004).

Child symptom severity and treatment outcome. The Youth Outcome Questionnaire (YOQ; Burlingame, Wells, Lambert, \& Cox, 2004) was used to track psychosocial treatment outcomes of youth participants. The $\mathrm{Y}-\mathrm{OQ}$ is a parent-report measure of treatment progress for children and adolescents (ages 4-17). It was designed to be utilized on a session-to-session basis with children and adolescents receiving psychological or psychiatric treatment. The $64 \mathrm{Y}-\mathrm{OQ}$ items were specifically constructed to assess the occurrence of observed behavior change. The Y-OQ yields a total score and six separate subscale scores with each of the subscales tapping various emotional and behavioral domains including: 1) Intrapersonal Distress, 2) Somatic, 3) Interpersonal Relations, 4) Critical items, 5) Social Problems, and 6) Behavioral Dysfunction. The total score is obtained by summing all six scales and is reflective of overall psychological distress the child or adolescent is currently experiencing. This total score is the best indicator of overall disturbance because of its high reliability and validity and has subsequently been recommended for tracking global change across treatment (Burglingame et al., 2004). Scores range from 0-180 with higher scores indicating greater psychopathology. Items for each domain are rated on a five-point Likert Scale (0 - never, 1 - rarely, 2 - sometimes, 3 - frequently, 4 almost always) with ratings based on how true each item is for the child over the previous seven 
days. Across treatment, reliable change has occurred if the individual's total score has changed at least 13 points (Burlingame et al., 2004).

The Y-OQ is a valid measure of psychosocial distress and psychotherapy outcome in children and adolescents (Burlingame et al. 2004) and possesses good internal and test-retest reliability, .95 and .83 respectively (Burlingame et al., 1996). Concurrent validity estimates have been computed between both Y-OQ total and subscale scores and the Achenbach's Child Behavior Checklist (CBCL) total and subscale scores (Achenbach, 1991) and the Conner's Parent Rating Scale total and subscale scores (CPRS-93, Conners, 1994). The highest correlation between these measures is found between the Y-OQ and the CBCL total scores $(r=.78)$. Validity coefficients range from .49 to .78 with the majority being in the high .50s to .60s (Burlingame et al., 1996). The Y-OQ has been shown to be sensitive to change in a variety of treatment settings (e.g. Berrett, 1999; Burlingame et al., 2001; Robinson, 2000; Mosier et al., 2001; Russell, 2003).

A self-report version has also been developed (Y-OQ-SR; Wells, Burlingame \& Rose, 2003) for youth ages 12 and above. Participants in the study meeting this age criteria completed the self-report version, which follows the same format as the Y-OQ. Like the Y-OQ, the Y-OQSR is a valid measure of psychosocial distress and psychotherapy outcome in adolescents. The Y-OQ-SR demonstrates good reliability and validity. The Y-OQ-SR total score demonstrates high internal consistency reliability coefficient alphas of .95, good test-retest reliability $(r=.89)$, and good concurrent validity with the CBCL $(r=0.83)$.

\section{Procedures}

The study employed a longitudinal design to examine the relation of putative mediator and moderator variables to treatment outcomes over time (until termination of therapy, or until 6 
months after intake). In determining timing and rate of measurement intervals, Laurenceau and colleagues (2007) recommend taking into account contextual characteristics such as the population being studied, as well as conceptualization of treatment effect and change. Measures to assess mediators and moderators should be taken over an appropriate time period and include multiple measurements to examine when these effects take place in psychotherapy and the degree of these effects (Laurenceau et al., 2007). For these reasons, study measures were given across 5 time periods. At intake, among other questionnaires, all parents completed the Youth Outcome Questionnaire (Y-OQ) and the Outcome Questionnaire (OQ-45). At intake, youth ages 12-17 completed the Y-OQ-SR. Parents and youth participants completed the same measures at 3 weeks after intake, and at 2-months, 4-months, and 6-months post-intake. Participants were given a \$10 gift card to a major retail discount store for participating in the intake data collection, and received a \$5 gift card for each subsequent participation (3 weeks, 2 months, 4 months, and 6 months after intake).

After intake, children and families began treatment. After initiation to treatment, therapy was assumed to occur on a weekly basis, but varied based on therapeutic goals, client needs, and therapist availability. A dose of treatment in this study was considered any therapeutic contact, including intake, individual therapy sessions and family sessions. Treatment implementation and dose varied according to child, diagnosis, and therapist. There were 38 service providers at Valley Mental Health with varying professional degrees and years of experience. Most of the children in the sample saw a clinical social worker $(n=96 ; 28.3 \%)$ or social worker $(n=103$; 30.4\%) while 34 (10\%) saw a psychologist, 49 (14.5\%) saw a social service worker, 43 (12.7\%) saw a licensed professional counselor, and 1 (0.3\%) saw a psychiatrist. 
Primary forms of intervention included individual and family psychotherapy, psychosocial skill-building groups, and medication management visits. Although a broad range of therapeutic approaches were used by clinicians in this traditional clinical setting, treatment could be generally characterized as following a family systems orientation. Specific emphases in the program included working with family members to change negative interaction patterns, teaching problem-solving and decision-making strategies, and exploring circumstances that led to current problems. Generally speaking, therapists in the program employed family therapy and cognitive strategies more frequently than psychodynamic or behavioral techniques.

Analysis

Hierarchical Linear Modeling (HLM) was used to test study hypotheses. HLM is a type of mixed model, which is often used with longitudinal data to examine inter-individual variability in change along with predictors or other covariates of interest that may affect the rate and shape of change (Laurenceau, Hayes \& Feldman, 2007; Singer \& Willett, 2003). In HLM analysis, data exist at more than one level (e.g. examining intra-individual level and interindividual level). This methodology allowed for examination of changes in the domains of parent distress, interpersonal relations, and social roles, as well as changes in youth symptomatology over the longitudinal data collection period. Further, hierarchical linear modeling revealed if and how a) changes in parent distress relate to changes in youth symptoms over the course of treatment, and b) changes in youth symptoms relate to changes in parent functioning.

Hierarchical linear modeling can be conceptualized as a two-stage model (Bryk \& Raudenbush, 1987; Laurenceau, et al., 2007: Singer \& Willett, 2003). Stage one examines individual change, as a combination of individual growth trajectories and random error (a regression model to capture intra-individual change). The second stage, models variability of 
these individual parameters around their average values (inter-individual variability)

(Laurenceau, et al., 2007). That is, interindividual differences in change and slope are examined as they relate to specific predictors of change (Singer \& Willett, 2003).

In examining moderation for my second hypothesis, if a pre-treatment between-subjects covariate (such as the initial level of parental psychological distress or interpersonal relations dissatisfaction) has an impact on the rate or shape of change for a psychotherapy outcome, it is considered a moderator of change (Laurenceau, et al., 2007).

In regards to my third hypothesis, the covariances between the intercepts and slopes of multiple dependent variables were examined using HLM, revealing if the relationships between parent functioning, interpersonal relationship satisfaction, and social role performance and changes in youth symptoms were related across time.

For these HLM analyses, treatment was modeled by weeks in treatment as opposed to number of therapy sessions due to variability in individual treatment dose and type of treatment received. That is, while treatment sessions were assumed to occur on a weekly basis, they varied based on client need, therapeutic goals, and therapist availability. Further, types of sessions varied across treatment including diagnostic sessions, individual sessions and family sessions. Thus, modeling treatment using weeks in treatment was more appropriate than using number of treatment sessions.

Within these analyses, repeated analyses were run for youth self-report and parent report of youth symptoms as dependent variables. This allowed for the examination of youth perceptions of their symptoms and change across treatment as they relate to parent functioning, as the observed relationship may be different from that of parent perceptions of youth symptoms and change. 
HLM holds many advantages as method of choice in examining change within longitudinal data. First, slope of change is variable across subjects and thus HLM does not require uniform data collection (Bryk \& Raudenbush, 1987). This is relevant given the nature of “real world” settings, where data collection varies based on client therapy attendance and timely completion of study measures. In this study, the flexibility in HLM for number and temporal spacing of data collection points is advantageous given the "real world" context in which data was obtained. HLM is also advantageous in that it is tolerant of missing data points and does not exclude an individual based on missingness of data (Bryk \& Raudenbush, 1987). This is relevant, again, given the context and setting of this study where missed therapy appointments and corresponding data collection points were evident in this sample and expected in such traditional clinical settings.

Alternative analyses using reliable change indices were ran to investigate study hypotheses. Research by Howard et al (1996) and Hansen, Lambert, and Forman (2002), have endorsed a dose-response model conceptualizing therapeutic change and evaluating treatment effectiveness. In this model, therapy functions as a 'dose' that is provided to the patient in varying degrees to achieve the desired 'response.' In applying the dose-response model, tracking the recovery or deterioration of clients in therapy facilitates the prediction of eventual outcome. Jacobson and Truax (1991) point to "clinical significance” as an important gauge of treatment effect when monitoring outcome. They developed a statistical index (known as the reliable change index, or RCI) to determine whether a change score on an outcome instrument is likely $(p<.05)$ to indicate actual or clinically significant change. With this in mind, Lambert (1998) developed algorithms based on a statistical index known as the reliable change index, or RCI, developed by Jacobson and Truax (1991). The RCI can be used to indicate whether a score on an 
outcome instrument is likely $(p<.05)$ to indicate clinically significant change. By applying this index to repeated outcome measures completed by patients, it is possible to identify patients who are benefiting from therapy, deriving no benefit from therapy, or deteriorating over the course of therapy. Using the reliable change indices for both the OQ-45 and the Y-OQ, as described earlier in the measures section, I was able to determine if significant and reliable change occurred for participants and examined the effect sizes of these changes. Further, I categorized participants according to the reliable change indices for parents who a) recovered b) reliably improved, c) evidenced no reliable change, and d) reliably deteriorated over the course of therapy.

In hypothesis 2a, I hypothesized that parent functioning would predict premature termination. No agreed upon operational definition of premature termination exists in the literature and this “definitional chaos” is a significant issue in studying child psychotherapy outcomes (e.g. Kazdin \& Mazurick, 1994). The definition of treatment dropouts have historically included individuals who are lost at various points across treatment, or is based on a time point in which they drop out (Kazdin \& Mazurick, 1994). Such inconsistencies call into question the reliability of findings regarding characteristics of individuals who drop out of treatment as different factors may affect and influence dropout at different time points across treatment (Kazdin \& Mazurick, 1994). In an effort to investigate all participants who prematurely dropped out in this study, premature dropout was defined as youth who presented to treatment with initial Y-OQ scores falling within clinical range and who withdrew from treatment during the course of psychotherapy prior to evidencing reliable symptom improvements, as measured by the RCI value for the Y-OQ. That is, premature termination was considered if the client discontinued treatment before significant symptom reduction and change occurred. To examine if parent functioning predicted premature termination, I conducted logistic regression as it is appropriate 
given the dichotomous nature of the dependent variable and continuous nature of the independent variable.

Results

Table 1 provides means and standard deviations of the OQ domains and Y-OQ scores at intake.

Table 1

Intake Means and Standard Deviations of OQ Domains and Y-OQ Scores

\begin{tabular}{lcc}
\hline & Mean & SD \\
\hline OQ Total & 62.4 & 23.4 \\
Symptom Distress & 36.3 & 15.3 \\
Interpersonal Relations & 15.6 & 6.4 \\
Social Role & 11 & 4.4 \\
Y-OQ & 87.8 & 35.5 \\
Y-OQ-SR & 71.3 & 35 \\
\hline
\end{tabular}

Hypothesis 1: Examining Whether Change Occurred in Parent Domains

I first examined the intercepts and slopes of the treatment outcome variables, specifically, the Y-OQ and Y-OQ-SR data collected over the course of treatment. To examine the rate of change of these variables, the Y-OQ and Y-OQ-SR data were fit to linear, quadratic and logarithmic models. Schwarz’s Bayesian Criterion (BIC), Akaike’s Information Criterion (AIC) and -2 Log Likelihood were used as indices of goodness of fit for each model. Results revealed that for the parent report of the Y-OQ, a logarithmic model fit the data best while a linear model fit better for the self-report version of the Y-OQ. For this reason, subsequent analyses used a logarithmic trajectory for the Y-OQ and a linear trajectory for the Y-OQ-SR. This indicates that parent perceived that their children's symptoms decreased over the course of treatment with 
change occurring most rapidly at the start of treatment and then tapering off by the end. Youth in the study, however, perceived a decrease in symptoms over time that followed a more steady, linear trajectory.

Table 2 provides the Intraclass Correlation Coefficients (ICC) for the parent dependent variables in this study. The ICC describes the portion of total variance in scores that are due to between-person differences and the portion of the within-person variance that is due to change across time. For example, the ICC for the OQ Total was .662, meaning that $66.2 \%$ of the total variance in OQ scores was due to between person differences and 33.8\% was due to within person variance or change over time.

Table 2

Intraclass Correlation Coefficients for OQ Variables

\begin{tabular}{ll}
\hline & ICC \\
\hline OQ Total & .662 \\
Symptom Distress & .691 \\
Interpersonal Relations & .672 \\
Social Role & .391 \\
\hline
\end{tabular}

To examine whether parent functioning improved in regards to symptom distress, interpersonal relations and social role satisfaction over the course of youth treatment, data were fit to linear, quadratic, and logarithmic models to examine best fit. Schwarz’s Bayesian Criterion (BIC), Akaike's Information Criterion (AIC) and -2 Log Likelihood were used as indices of goodness of fit for each model. Results revealed that for OQ Total and Symptom Distress domain, a linear model fit slightly better but a chi-square difference test indicated nonsignificance at the .05 level between the linear model and logarithmic model. For the 
Interpersonal Relations and Social Role domains, a logarithmic model fit slightly better, but was also not significantly better than a linear model at the .05 level. Precedents in the literature suggest that a logarithmic trajectory is a good fit for treatment progress and recovery (e.g. Finch, Lambert, \& Schaalje, 2001; Lambert, Whipple, Bishop, Vermeersch, Gray \& Finch, 2002; Spielmans, Masters \& Lambert, 2006). Consequently, a logarithmic trajectory was used for this and subsequent analyses of OQ domains.

Age was also tested as a covariate for all OQ domains and Y-OQ parent and self-report in efforts to examine whether the age of the child receiving treatment was differentially related to changes in parent and youth symptoms. No significant interaction was found for age on any of the domains examined, indicating that age of the child did not significantly influence change in youth or parent symptoms.

Table 3 presents the results of analyses examining OQ domain change trajectories. The fixed effects indicate the average change trajectory of the entire sample and the random effects indicate the individual variation in change trajectories. 
Table 3

Fixed Effect Estimates, Random Variance, and Standard Errors for OQ Domain and $O Q$ Total Change Trajectories

\begin{tabular}{|c|c|c|c|c|}
\hline & & Estimate & SE & $p$ \\
\hline \multicolumn{5}{|l|}{ Symptom Distress } \\
\hline \multirow[t]{2}{*}{ Fixed Effects } & Intercept & 36.1 & .942 & $<.001$ \\
\hline & LNWKS & -.701 & .305 & .023 \\
\hline \multirow[t]{2}{*}{ Random Effects } & Intercept & 166.98 & 21.1 & $<.001$ \\
\hline & Slope & 1.678 & 2.11 & .426 \\
\hline \multicolumn{5}{|c|}{ Interpersonal Relations } \\
\hline \multirow[t]{2}{*}{ Fixed Effects } & Intercept & 15.45 & .402 & $<.001$ \\
\hline & LNWKS & -.121 & .14 & .390 \\
\hline \multirow[t]{2}{*}{ Random Effects } & Intercept & 28.31 & 3.86 & $<.001$ \\
\hline & Slope & .436 & .451 & .334 \\
\hline \multicolumn{5}{|l|}{ Social Role } \\
\hline \multirow[t]{2}{*}{ Fixed Effects } & Intercept & 11.02 & .275 & $<.001$ \\
\hline & LNWKS & -.298 & .128 & .021 \\
\hline \multirow[t]{2}{*}{ Random Effects } & Intercept & 7.75 & 2.05 & $<.001$ \\
\hline & Slope & .410 & .40 & .306 \\
\hline \multicolumn{5}{|l|}{ OQ Total } \\
\hline \multirow[t]{2}{*}{ Fixed Effects } & Intercept & 62.30 & 1.472 & $<.001$ \\
\hline & LNWKS & -1.147 & .519 & .029 \\
\hline \multirow{2}{*}{$\begin{array}{r}\text { Random } \\
\text { Effects }\end{array}$} & Intercept & 371.40 & 50.62 & $<.001$ \\
\hline & Slope & 6.556 & 5.952 & .271 \\
\hline
\end{tabular}

Note. The LNWKS effect estimate refers to the amount of increase in OQ domain or total score per one unit increase in the natural log of the number of weeks in treatment. 
Results indicate that the average change trajectory for the OQ total score was significant $(p<.05)$, and for every one unit increase in the natural log of the number of weeks a parent's child was in treatment (LNWKS), their OQ total score decreased by a little more than 1 point. Random variance in change trajectory, as indicated by the random intercept and slope terms, reflects the amount of individual variation in intake OQ scores and rate of change in OQ scores. In regards to the OQ Total score, although significant individual variation was observed in intake OQ scores, there did not appear to be significant individual variation in the rate of change. Figure 1 illustrates the average trajectory for the total OQ.

Figure 1

Average $O Q$ Trajectory

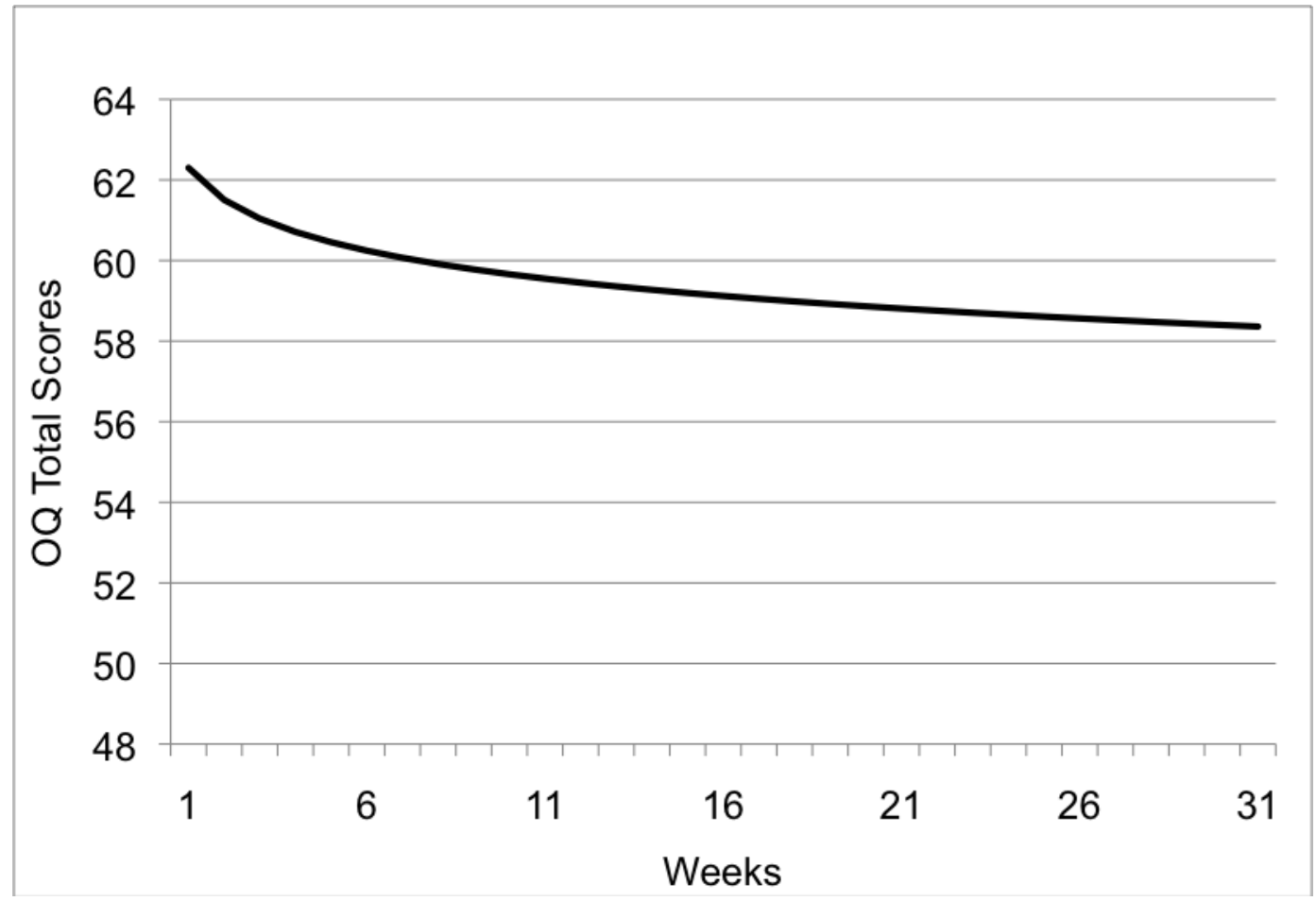


Similarly, fixed effects for the domain scores of the OQ revealed that the average trajectories for Symptom Distress and Social Role were significant, indicating an overall decrease in scores over time. Interpersonal Relations did not yield significant fixed effects, indicating a relatively flat trajectory. Significant random effects were not observed for the slopes of these domains, indicating there was not significant individual variation in rate of change.

Figures 2, 3, and 4 illustrate the average trajectories for these domains.

Figure 2

Average Symptom Distress Trajectory

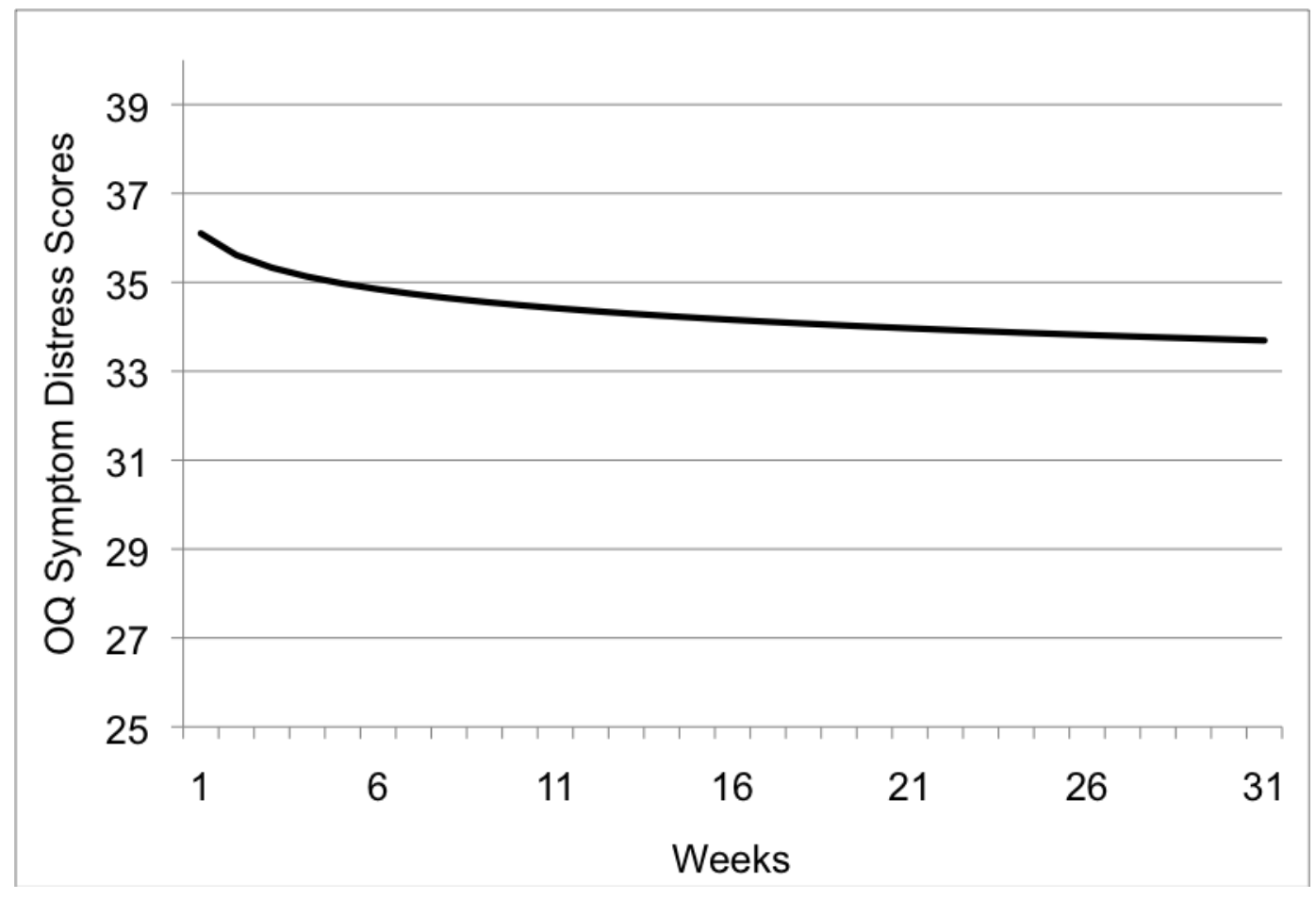


Figure 3

Average Interpersonal Relations Trajectory

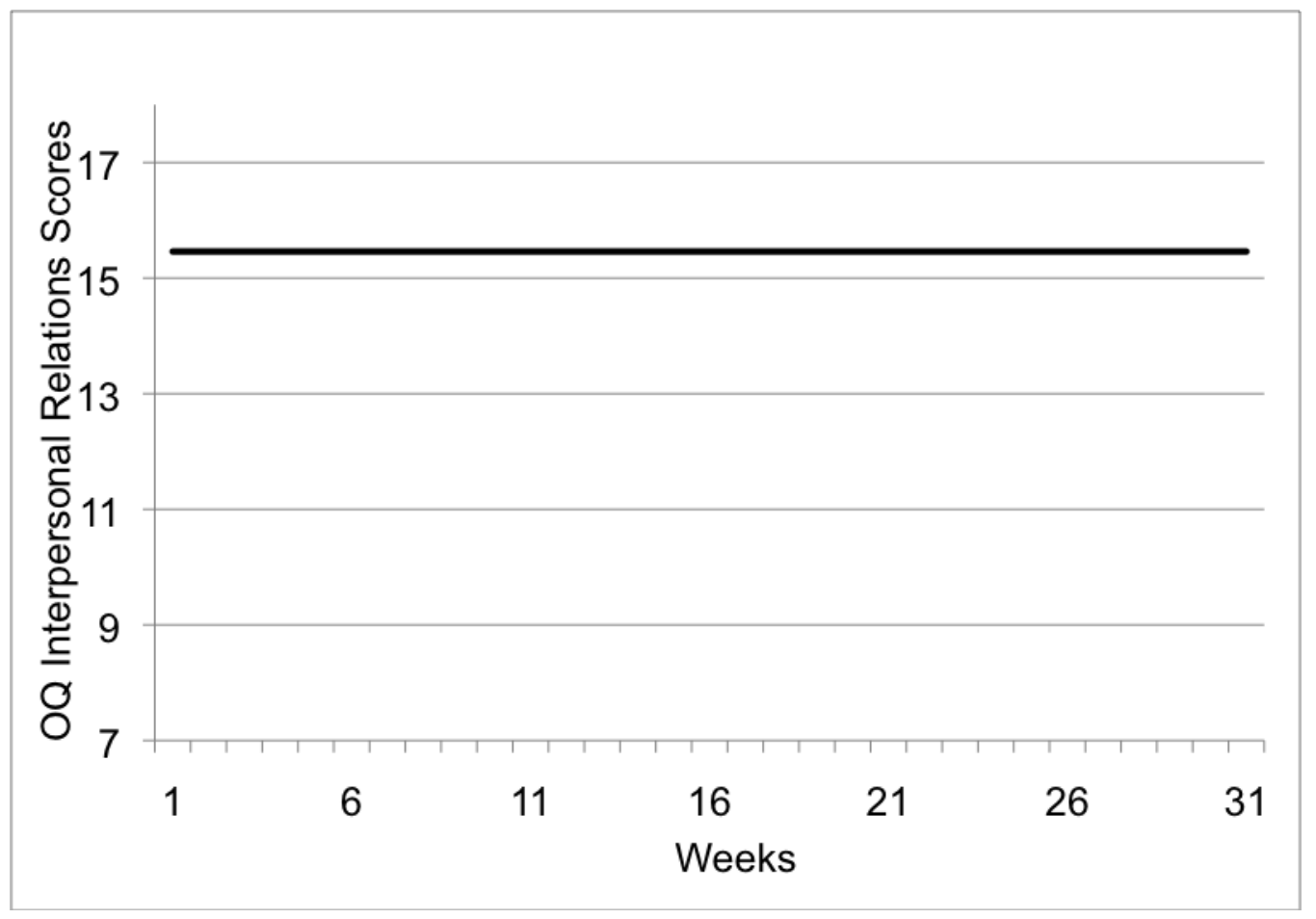


Figure 4

Average Social Role Trajectory

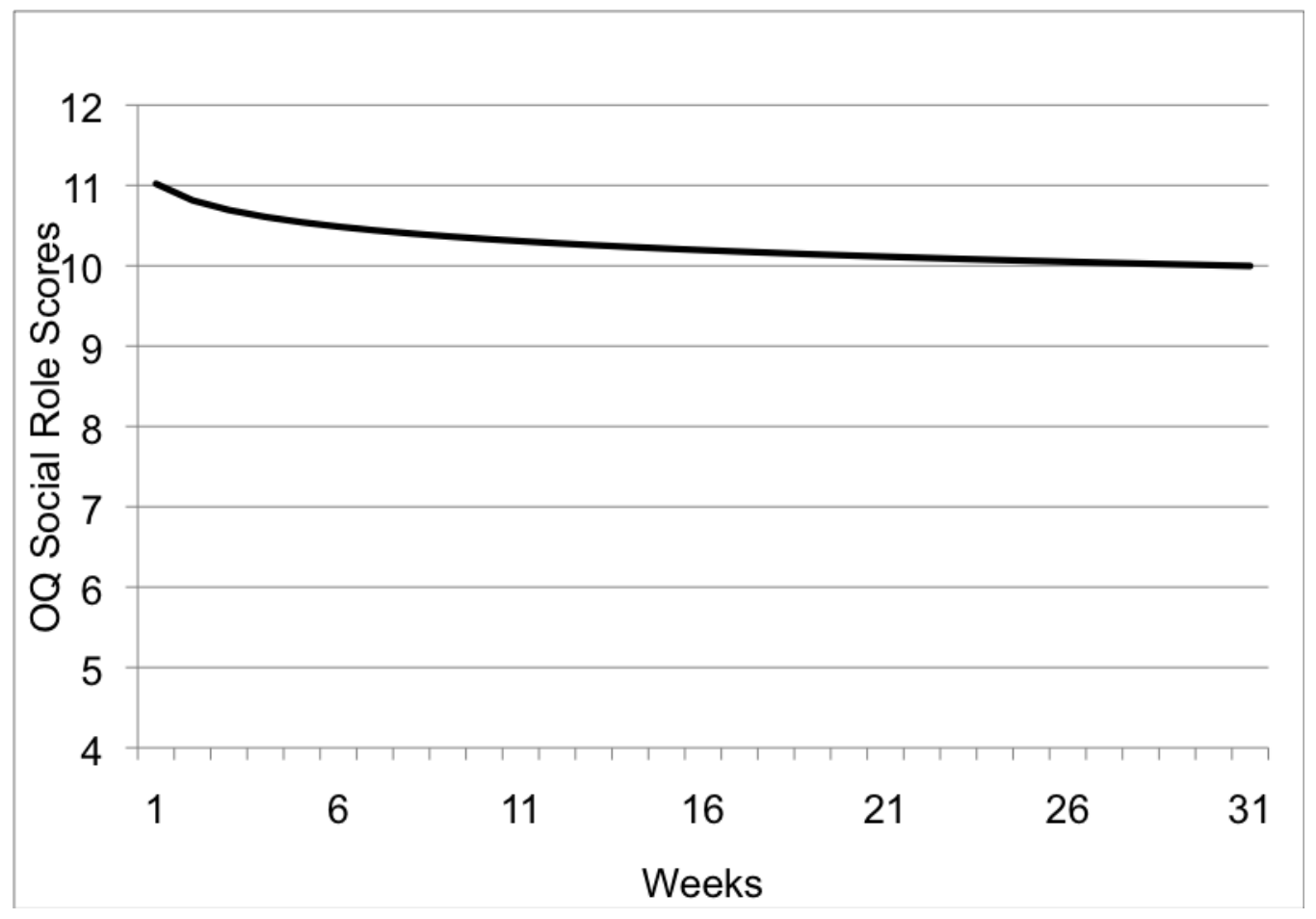

As described in the Method section, cutoff scores and reliable change indices were used to examine clinically significant change in parent OQ scores. Change scores were calculated by taking the parent's last OQ-45 total and domain scores and subtracting them from the intake OQ45 total and domain scores. Then change scores were categorized into 4 categories: Recovered, Reliably Improved, No Reliable Change, and Deteriorated. Change scores met criteria for Recovered if 1) the first score fell above the cutoff score and the final score fell below the cutoff score and 2) the score changed beyond the specified RCI value. A case was categorized as Reliably Improved if the change score fell into the clinically significant change category but did not fall below the cutoff score, or their initial score did not fall into the clinically significant range. The No Reliable Change category was applied to cases where scores did not meet requirements for clinically significant change. The Deteriorated category was applied to cases 
where change scores fell in the clinically significant change range but demonstrated an exacerbation of symptoms, or a significant increase from initial score to final score matching the RCI value. The results of these analyses are presented in Table 4.

Table 4

Change Categories for $O Q$ Scores

\begin{tabular}{|c|c|c|c|c|c|}
\hline & & OQ Total & $\begin{array}{l}\text { Symptom } \\
\text { Distress }\end{array}$ & $\begin{array}{c}\text { Interpersonal } \\
\text { Relations }\end{array}$ & Social Role \\
\hline \multicolumn{6}{|l|}{ Recovered } \\
\hline & Frequency & 20 & 24 & 9 & 15 \\
\hline & Percent & $12 \%$ & $13 \%$ & $5 \%$ & $8 \%$ \\
\hline \multicolumn{6}{|l|}{$\begin{array}{l}\text { Reliably } \\
\text { Improved }\end{array}$} \\
\hline & Frequency & 31 & 23 & 9 & 9 \\
\hline & Percent & $18 \%$ & $12 \%$ & $5 \%$ & $5 \%$ \\
\hline \multicolumn{6}{|l|}{$\begin{array}{l}\text { No Reliable } \\
\text { Change }\end{array}$} \\
\hline & Frequency & 93 & 119 & 150 & 148 \\
\hline & Percent & $53 \%$ & $63 \%$ & $83 \%$ & $81 \%$ \\
\hline \multicolumn{6}{|l|}{ Deteriorated } \\
\hline & Frequency & 30 & 24 & 13 & 11 \\
\hline & Percent & $17 \%$ & $13 \%$ & $7 \%$ & $6 \%$ \\
\hline
\end{tabular}

Results indicated that the majority of cases fell in the No Reliable Change category across the symptom domains as well as total scores. However, a significant portion of parents did report significant reliable improvement in their overall symptom profile as well as across the various domains. Further, a significant portion also fell into the Recovered category in all these areas.

Subsequent analyses were performed to further examine the Reliable Change Indices for this study sample. Specifically, the sample was divided into a Clinical Sample and Subclinical Sample and RCI values were calculated for these subsamples. The Clinical Sample was defined 
as parents whose intake OQ scores fell above the cutoff score of 64, indicating presence of significant distress. The Subclinical Sample was comprised of those parents whose intake OQ scores fell below the cutoff score, demonstrating a relative lack of psychological distress. In this sample, 111 (46.8\%) of parents in the total sample met criteria for the Clinical Sample and 126 (53.2\%) met criteria for the Subclinical Sample. Results for the Clinical Sample and the Subclinical Sample are presented in Table 5 and Table 6 respectively.

Table 5

Change Categories for Clinical Sample OQ Scores

\begin{tabular}{|c|c|c|c|c|c|}
\hline & & OQ Total & $\begin{array}{l}\text { Symptom } \\
\text { Distress }\end{array}$ & $\begin{array}{c}\text { Interpersonal } \\
\text { Relations }\end{array}$ & Social Role \\
\hline \multicolumn{6}{|l|}{ Recovered } \\
\hline & Frequency & 20 & 24 & 9 & 15 \\
\hline & Percent & $25 \%$ & $25 \%$ & $10 \%$ & $25 \%$ \\
\hline \multicolumn{6}{|l|}{$\begin{array}{l}\text { Reliably } \\
\text { Improved }\end{array}$} \\
\hline & Frequency & 15 & 13 & 2 & 1 \\
\hline & Percent & $19 \%$ & $14 \%$ & $2 \%$ & $2 \%$ \\
\hline \multicolumn{6}{|l|}{$\begin{array}{l}\text { No Reliable } \\
\text { Change }\end{array}$} \\
\hline & Frequency & 35 & 51 & 75 & 44 \\
\hline & Percent & $44 \%$ & $54 \%$ & $84 \%$ & $73 \%$ \\
\hline \multicolumn{6}{|l|}{ Deteriorated } \\
\hline & Frequency & 10 & 7 & 3 & 0 \\
\hline & Percent & $13 \%$ & $7 \%$ & $3 \%$ & - \\
\hline
\end{tabular}


Table 6

Change Categories for Subclinical Sample OQ Scores

\begin{tabular}{|c|c|c|c|c|c|}
\hline & & OQ Total & $\begin{array}{l}\text { Symptom } \\
\text { Distress }\end{array}$ & $\begin{array}{c}\text { Interpersonal } \\
\text { Relations }\end{array}$ & Social Role \\
\hline \multicolumn{6}{|l|}{$\begin{array}{l}\text { Reliably } \\
\text { Improved }\end{array}$} \\
\hline & Frequency & 16 & 10 & 7 & 8 \\
\hline & Percent & $17 \%$ & $11 \%$ & $8 \%$ & $7 \%$ \\
\hline \multicolumn{6}{|l|}{$\begin{array}{l}\text { No Reliable } \\
\text { Change }\end{array}$} \\
\hline & Frequency & 58 & 68 & 75 & 104 \\
\hline & Percent & $62 \%$ & $72 \%$ & $82 \%$ & $85 \%$ \\
\hline \multicolumn{6}{|l|}{ Deteriorated } \\
\hline & Frequency & 20 & 17 & 10 & 11 \\
\hline & Percent & $21 \%$ & $18 \%$ & $11 \%$ & $9 \%$ \\
\hline
\end{tabular}

Results from the Clinical Sample analyses reveal that a greater proportion fell into the Recovered and Reliably Improved categories across the OQ Total and domains as compared to the combined sample. Further, fewer fell into the Deteriorated category compared to the combined sample. In contrast, these analyses reveal that more participants in the Subclinical category fell into the No Reliable Change and Deteriorated categories across the OQ Total and domains than the combined sample, with the exception of the Interpersonal Relations domain. Thus, in general, parents who demonstrated significant psychological distress at intake, tended to perceive greater personal improvement in symptoms across the course of their children receiving psychotherapy compared to parents who upon initiation of youth psychotherapy did not indicate significant distress.

Hypothesis 2: Intake OQ Scores Predicting Changes in Y-OQ Scores and Intake Y-OQ Scores Predicting Changes in OQ Scores.

Intake OQ scores were tested as predictors of Y-OQ intake scores and symptom change over the course of treatment. Results of the fixed effects of parent OQ intake scores predicting 
Y-OQ scores are presented in Table 7. Results of fixed effects of OQ scores predicting Y-OQ-SR scores are presented in Table 8. Based on the results from preliminary analyses, parent report of youth symptom changes were fit to a logarithmic function as indicated by LNWKS (natural log transformation of the number of weeks in treatment) and youth report of youth symptom changes were fit to a linear function as indicated by WEEKS. 
Table 7

Estimates and Standard Errors for Intake OQ Scores Predicting Y-OQ Intake Scores and Change

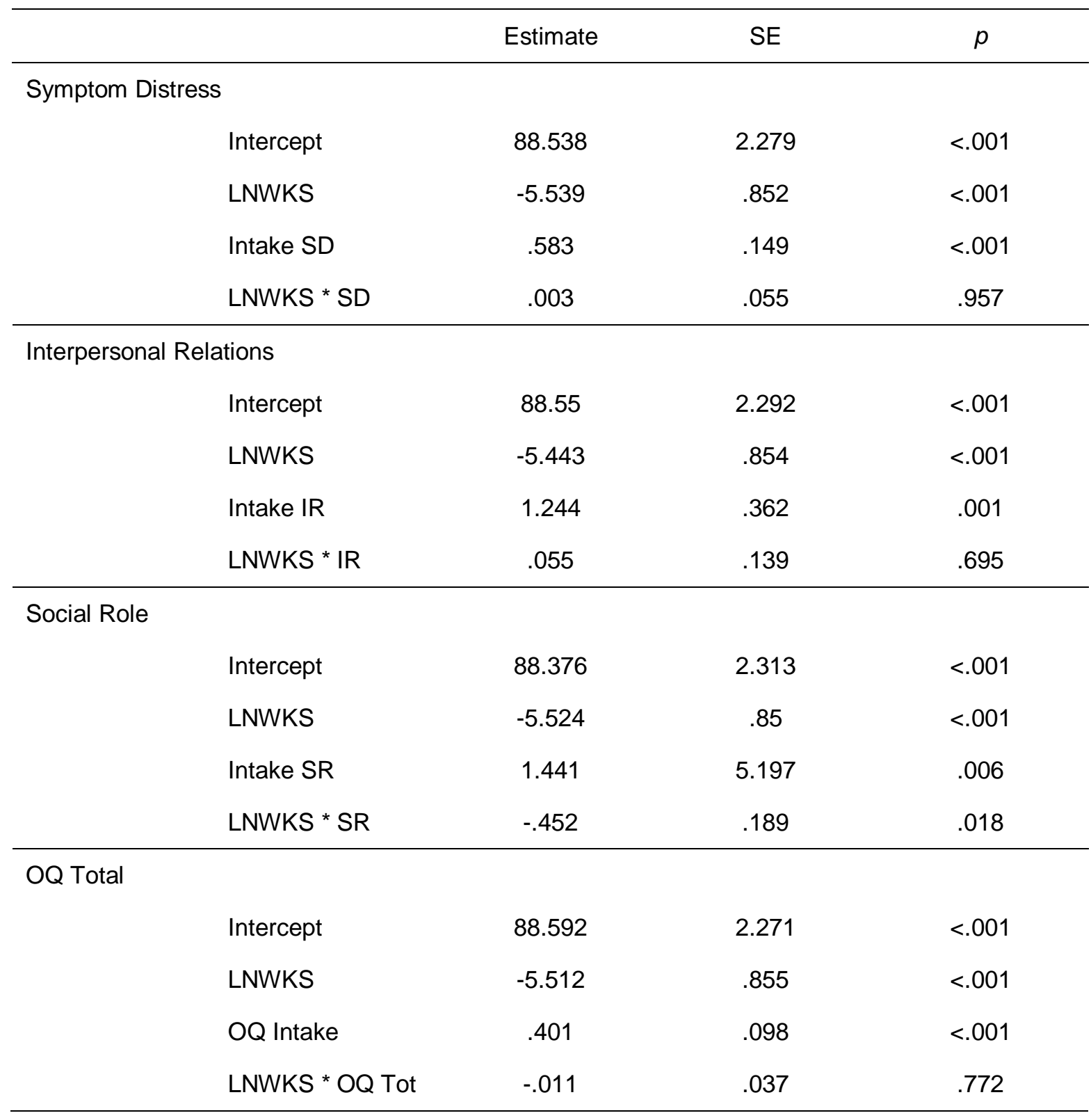

Results from Table 7 indicate that all intake OQ scores significantly predicted intake YOQ scores, evidencing a significant relationship at the initiation of treatment, as hypothesized. Parent Social Role performance was the only significant predictor (at intake) of change in Y-OQ 
scores over the course of treatment. Figure 5 illustrates the observed relationship between intake Social Role performance and changes in youth symptoms. As can be observed from the figure, the more dissatisfied parents were with their social role performance (higher scores) at intake, the more change they reported in their youths' symptoms. In the figure, Y-OQ trajectories are illustrated using intake Social Role scores approximately one standard deviation above and below the mean for this sample.

Figure 5

Social Role Performance at Intake Predicting Changes in Y-OQ Scores

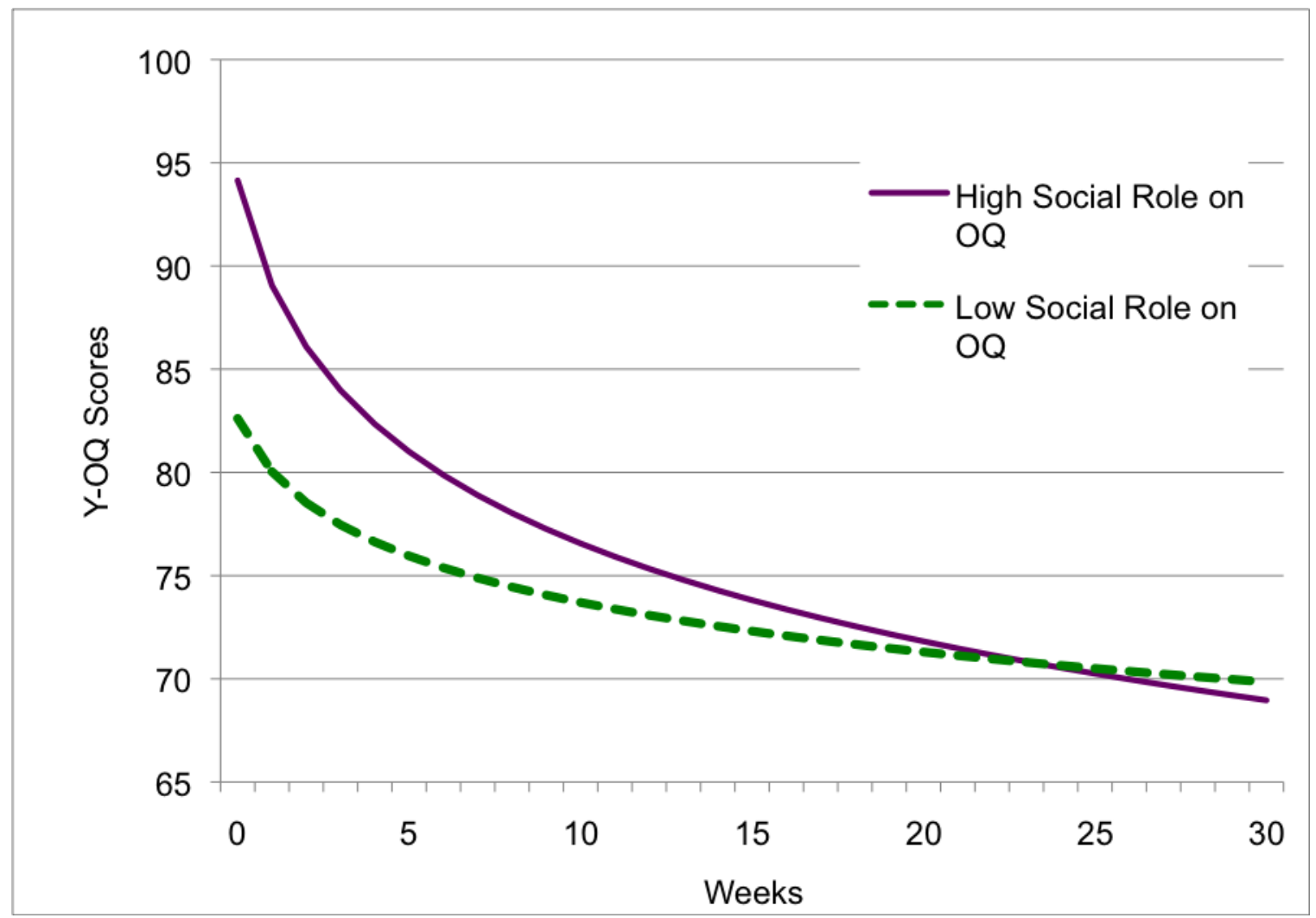


Table 8

Estimates and Standard Errors for Intake OQ Scores Predicting Y-OQ-SR Intake Scores and Change

\begin{tabular}{|c|c|c|c|}
\hline & Estimate & SE & $p$ \\
\hline \multicolumn{4}{|l|}{ Symptom Distress } \\
\hline Intercept & 74.418 & 3.537 & $<.001$ \\
\hline WEEKS & -.495 & .195 & .015 \\
\hline SD Intake & .489 & .233 & .038 \\
\hline WEEKS * SD & -.0122 & .013 & .350 \\
\hline \multicolumn{4}{|l|}{ Interpersonal Relations } \\
\hline Intercept & 74.693 & 3.563 & $<.001$ \\
\hline WEEKS & -.597 & .201 & .005 \\
\hline IR Intake & 1.011 & .565 & .076 \\
\hline WEEKS * IR & -.063 & .031 & .049 \\
\hline \multicolumn{4}{|l|}{ Social Role } \\
\hline Intercept & 74.616 & 3.582 & $<.001$ \\
\hline WEEKS & -.608 & .205 & .005 \\
\hline SR Intake & 1.214 & .802 & .133 \\
\hline WEEKS * SR & -.094 & .051 & .071 \\
\hline \multicolumn{4}{|l|}{ OQ Total } \\
\hline Intercept & 74.764 & 3.541 & $<.001$ \\
\hline WEEKS & -.557 & .201 & .008 \\
\hline OQ Intake & .341 & .155 & .029 \\
\hline WEEKS * OQ & -.014 & .009 & .127 \\
\hline
\end{tabular}

Results from Table 8 indicate that Symptom Distress and OQ total scores at intake significantly predicted Y-OQ-SR intake scores, indicating a significant relationship between these domains at initiation of therapy as hypothesized. Interpersonal Relations was the only 
significant predictor (at intake) of change in Y-OQ-SR scores over the course of treatment. Further, parent Social Role performance at intake approached significance as a predictor of changes in self-reported youth symptoms over the course of treatment. Figure 6 illustrates the observed relationship between intake Interpersonal Relations and Y-OQ-SR scores over the course of treatment. As can be observed, youth of parents who reported higher distress in their interpersonal relations (high IR scores) reported greater change in their self-reported symptoms. In the figure, Y-OQ-SR trajectories are illustrated using intake Interpersonal Relations scores approximately one standard deviation above and below the mean for this sample.

Figure 6

Interpersonal Relations at Intake Predicting Changes in Y-OQ-SR Scores

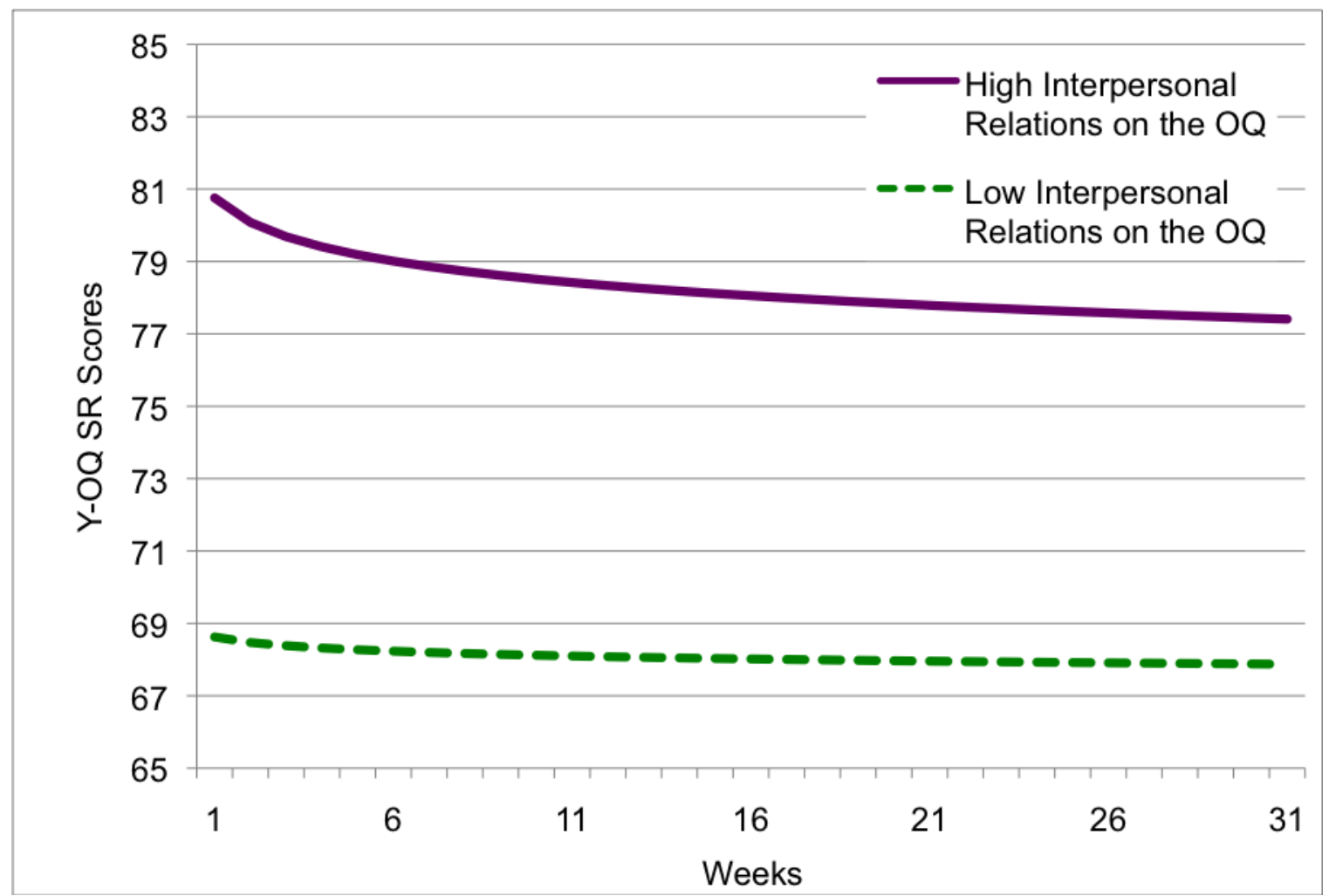

In hypothesis 2a, I also hypothesized that parent functioning would predict premature termination. As mentioned previously, premature termination was defined as youth who 
presented to treatment with initial Y-OQ scores falling in the clinical range and who dropped out of treatment prior to demonstrating reliable symptom improvement, as measured by the RCI value for the Y-OQ. That is, a case was identified as prematurely terminated if the client discontinued treatment before significant symptom reduction and change occurred. Because the dependent variable in this hypothesis (dropout) was dichotomous and the independent variable was continuous, logistic regression was used to test this hypothesis. In this sample, 83 cases (23\%) were identified as premature dropouts from treatment. The OQ domains of Social Role, Interpersonal Relations, and Symptom Distress were entered into the equation predicting premature dropout. Results of this analysis are presented in Table 9. Results indicated that none of the OQ domains significantly predicted treatment dropout.

Table 9

Intake OQ Domains Predicting Premature Termination

\begin{tabular}{lcccc}
\hline & B & S.E. & $p$ & Exp(B) \\
\hline Social Role & .093 & .086 & .280 & 1.098 \\
$\begin{array}{l}\text { Interpersonal } \\
\text { Relations }\end{array}$ & -.061 & .057 & .289 & .941 \\
$\begin{array}{l}\text { Symptom } \\
\text { Distress }\end{array}$ & -.010 & .025 & .684 & .990 \\
\hline
\end{tabular}

In hypothesis 2b, I hypothesized that youth symptoms at intake would significantly predict parent OQ scores at intake and rate of change in OQ scores. Intake scores for the Y-OQ and Y-OQ-SR were tested as predictors of parent Symptom Distress, Interpersonal Relations, Social Role performance, and OQ total scores at intake and rate of change in these variables over 
the course of treatment. Results of the fixed effects of Y-OQ and Y-OQ-SR intake scores as predictors are presented in Tables 10 and 11, respectively.

Table 10

Estimates and Standard Errors for Intake Y-OQ Scores Predicting OQ Intake Scores and Change

\begin{tabular}{|c|c|c|c|}
\hline & Estimate & SE & $p$ \\
\hline \multicolumn{4}{|l|}{ Symptom Distress } \\
\hline Intercept & 35.875 & 1.458 & $<.001$ \\
\hline LNWKS & -.151 & .455 & .746 \\
\hline Y-OQ & .073 & .038 & .060 \\
\hline LNWKS * Y-OQ & .010 & .013 & .435 \\
\hline \multicolumn{4}{|l|}{ Interpersonal Relations } \\
\hline Intercept & 14.821 & .602 & $<.001$ \\
\hline LNWKS & .238 & .189 & .214 \\
\hline Y-OQ & .038 & .016 & .017 \\
\hline LNWKS * Y-OQ & .008 & .005 & .144 \\
\hline \multicolumn{4}{|l|}{ Social Role } \\
\hline Intercept & 10.629 & .427 & $<.001$ \\
\hline LNWKS & -.122 & .179 & .498 \\
\hline Y-OQ & .012 & .011 & .270 \\
\hline LNWKS * Y-OQ & -.000 & .005 & .960 \\
\hline \multicolumn{4}{|l|}{ OQ Total } \\
\hline Intercept & 60.421 & 2.224 & $<.001$ \\
\hline LNWKS & .222 & .766 & .773 \\
\hline Y-OQ & .141 & .058 & .017 \\
\hline LNWKS * Y-OQ & .017 & .021 & .410 \\
\hline
\end{tabular}


Table 11

Estimates and Standard Errors for Intake Y-OQ-SR Scores Predicting OQ Intake Scores and Change

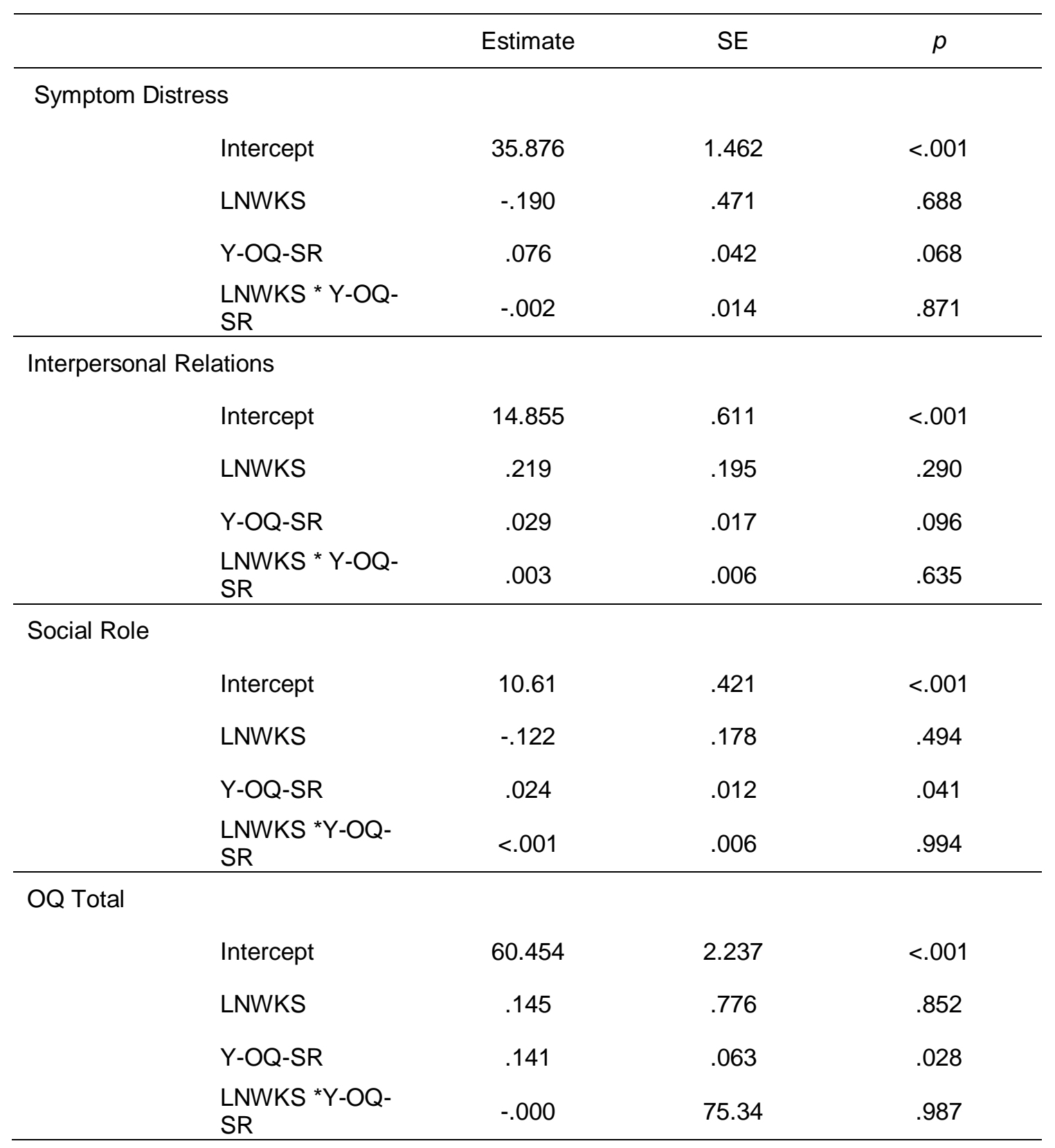

Results from Tables 10 and 11 indicate that parent rated youth symptoms at intake significantly predicted parent intake Interpersonal Relations and OQ total scores, and youth rated symptoms at intake significantly predicted parent intake Social Role performance and OQ total 
scores. However, contrary to hypotheses, youth symptoms at intake, as reported by both parents and youth, did not significantly predict any changes in parent symptoms as measured by OQ scores over the course of treatment.

Hypothesis 3: Relationship Between Changes in OQ Scores and Changes in Youth Symptoms In hypothesis 3, I hypothesized that changes in OQ scores over the course of treatment would be associated with changes in youth symptoms. To examine these associations, covariances were examined between the slopes of parent OQ scores and the slopes of Y-OQ and Y-OQ-SR scores. Results of these analyses are presented below in Tables 12 and 13.

\section{Table 12}

Covariances and Standard Errors for $O Q * Y-O Q$ Slope Interactions

\begin{tabular}{lccc}
\hline & Estimate & SE & $p$ \\
\hline $\begin{array}{l}\text { Symptom distress * } \\
\text { Y-OQ }\end{array}$ & .0248 & .0167 & .135 \\
$\begin{array}{l}\text { Interpersonal } \\
\text { Relations * } \\
\text { Y-OQ }\end{array}$ & .0310 & .0181 & .086 \\
$\begin{array}{l}\text { Social Role * } \\
\text { Y-OQ } \\
\text { OQ Total * Y-OQ }\end{array}$ & .1112 & .0309 & .000 \\
\hline
\end{tabular}


Table 13

Covariances and Standard Errors for the $O Q * Y-O Q-S R$ Slope Interactions

\begin{tabular}{lccc} 
& Estimate & SE & $p$ \\
\hline $\begin{array}{l}\text { Symptom distress * } \\
\text { Y-OQ-SR }\end{array}$ & .0255 & .0140 & .070 \\
$\begin{array}{l}\text { Interpersonal } \\
\text { Relations * } \\
\text { Y-OQ-SR }\end{array}$ & .0226 & .0138 & .101 \\
$\begin{array}{l}\text { Social Role * } \\
\text { Y-OQ-SR } \\
\text { OQ Total * Y-OQ-SR }\end{array}$ & .0889 & .0296 & .003 \\
\hline
\end{tabular}

The covariance estimates presented in Tables 12 and 13 indicate the degree to which the slopes of the OQ domains and the Y-OQ scores covary at the individual level. The results presented above partially support my third hypothesis as a significant relationship was found in the progression of symptom change between both the parent and self-report versions of the YOQ and the OQ Total score and Social Role domain. In other words, changes in OQ Total scores and Social Role scores were associated with changes in Y-OQ scores (both parent- and youth self-report) over the course of treatment. Contrary to hypothesis, no significant relationship was found between the slopes of Y-OQ scores and the Symptom Distress or Interpersonal Relations domains.

\section{Discussion}

The chief purposes of this study were as follows: 1) to examine whether three aspects of parent functioning — symptom distress, interpersonal relationships, and social role performance-changed over the course of the youth treatment, 2) to examine how these factors as measured at intake predicted youth symptoms and progress in psychotherapy and 3) to examine whether changes in these parent factors over the course of youth psychotherapy were 
associated with changes in youth symptoms. The results of this study provide support for several of the study hypotheses. The most significant findings of this study are that specific parent domains improved over the course of treatment, and that the progression of changes in specific parent domains were related to the progression of changes in youth scores across treatment.

The findings of this study supported many of the initial hypotheses. In reference to my first hypothesis, overall parent symptomatology as measured by the OQ total, Symptom Distress and Social Role domains improved significantly over the course the course of treatment. Further, using the Reliable Change Indices, results indicated that parents who demonstrated greater distress at the initiation of psychotherapy treatment for their children demonstrated greater change and improvement in symptoms across treatment compared to parents who did not indicate significant psychological distress at intake. These finding support the notion that parents indirectly benefited from their children receiving therapeutic services. This is particularly relevant as these results suggest that parents who demonstrated significant distress indirectly received more benefit from their children receiving therapeutic services, as indicated by greater improvement in symptoms. These are important findings that build on the findings of Kazdin and Wassel (2000) and emphasizes that the scope and benefits of community-based mental health therapy for youth extends to parents. These findings also underscore the potential benefits of youth treatment for improving family functioning and maintaining therapeutic changes (e.g. Kazdin, 2004).

Study results largely supported the second hypothesis that OQ domains would be significant predictors of youth intake scores and changes in youth scores over the course of treatment. Specifically, a significant relationship was found between all parent OQ domains and parent rated youth scores at intake. Further, overall parent symptomatology and the domain of 
Symptom Distress were significantly related to youth rated intake scores. These findings provide further evidence for the relationship between parent functioning and youth functioning (e.g. Downey \& Coyne, 1990; Hammen et al, 2004; Kazdin, 1994, 2003, 2004; Reyno \& McGrath, 2006). Similarly, although I expected that parent symptom distress would be a significant predictor of changes in youth scores over the course of treatment, parent Social Role performance and Interpersonal Relations turned out to be the most significant predictors. Parent rated Social Role performance was a significant predictor of changes in youth scores over the course of treatment. In answering the OQ-45 Social Role domain questions, instructions indicate that participants should interpret questions regarding work as employment, school, housework, volunteer work and so forth. Because the vast majority of this sample was comprised of stay-at-home mothers, it was assumed that the mothers' interpretations of questions regarding work were interpreted in regards to their roles as mothers and homemakers. This finding suggests that parent (particularly mothers') level of dissatisfaction, distress, and inadequacy in relations to their family roles is significantly related to treatment progress for their children. This finding makes conceptual sense. Parents who are more satisfied and feel a sense of adequacy in their position as a parent may be able to influence their child's treatment in a variety of ways. For instance, such a parent would likely have more positive interactions with their children, may be able to reinforce and implement skills taught in treatment for their children, and may be more emotionally and physically available to parent, which may be directly related to treatment progress in their children. Such a relationship was observed by Hemphill and Littlefield (2006) and Calam and colleagues (2002), demonstrating the importance of positive parent-child interactions and therapy outcomes as reviewed previously. 
Parent scores on the Interpersonal Relations domain at intake significantly predicted selfreported changes in youth scores over the course of treatment. This finding is consistent with the literature reviewed previously and supports the notion that difficulties in interpersonal relationships are associated with youth psychotherapy outcomes (e.g. Calam et al., 2002; Hamphill \& Littlefield, 2006; Kazdin, 1995). However, this relationship was not significant when youth scores were measured by parent report. A potential explanation for this finding is that the youth self-report version is limited to youth ages 12 and older, whereas the total sample of youth was included in the Y-OQ. In this sample, this finding might suggest a stronger relationship between parent difficulties in interpersonal relationships and psychotherapy outcomes for older youth. Various studies observe such a significant relationship between parent interpersonal difficulties and adolescent psychosocial adjustment (e.g. Forehand, Wierson, Thomas \& Fauber, 1991; Krishnakumar, Buehler \& Barber, 2003; Li, 1997).

Finally, over the course of treatment, significant covariations between the trajectories in OQ total and the domain of Social Role performance were found with both parent and youth report of symptoms on the Y-OQ. This indicates a significant relationship between the progression of overall parent symptoms, Social Role performance scores and youth progress in therapy. The significant finding between the trajectories of parents’ social role performance and youth treatment outcomes makes sense as a child's treatment outcome is likely related to a parent's sense of satisfaction and adequacy in their role as a parent. Further, parent changes in their OQ total score, as an overall measure of parent psychological distress, significantly covaried with changes in youth scores over the course of treatment. While OQ total at intake did not significantly predict changes in youth scores, the observed relationship between the progression of total parent psychological distress and progression of changes in youth symptoms 
in treatment is consistent with the literature reviewed previously (e.g. Berman et al., 2000; Brent et al., 1998; Cobham et al., 1998; Kazdin \& Wassel, 1998; Reyno \& McGrath, 2006; WebsterStratton \& Hammond, 1990) indicating a significant and important relationship between changes in parent symptomatology and youth progress in therapy. Although this was not a direct test of mediation, these results are consistent with the notion that parent Social Role performance and overall symptomatology may be potential mediators in youth psychotherapy.

A number of hypotheses were not supported by study results. First, contrary to hypotheses, parent Symptom Distress did not influence youth treatment outcome nor did changes in parent Symptom Distress covary significantly with youth symptom progression. This is intriguing given the empirical evidence of a relationship between parent psychopathology and youth treatment outcomes (e.g. Berman et al., 2000; Brent et al., 1998; Cobham et al., 1998; Kazdin \& Wassel, 1998; Reyno \& McGrath, 2006; Webster-Stratton \& Hammond, 1990). Although this relationship was not observed in these study findings, the observation that changes in overall parent symptomatology, as measured by the OQ total scores, covaried significantly with youth progress in therapy supports my hypothesis that changes in parent symptomatology are related to youth treatment outcomes.

Also contrary to hypotheses, OQ domains at intake did not predict premature termination. However, while the literature does suggest a relationship between parent functioning and youth treatment dropout (e.g. Calam et al. 2002) this relationship is not strongly established in the literature. For example, Kazdin and Wassel (1998) found that parent symptomatology and functioning did not relate to treatment drop-out, but was rather related to treatment outcomes, similar to the findings in this study. Consequently, the relation between parent functioning and youth treatment dropout warrants further inquiry. 
Finally, youth scores at intake did not predict changes in parent OQ domains over the course of treatment. Although I had expected to find a relationship here, the literature more strongly supports the impact of parent functioning on youth outcomes in therapy (e.g. Berman et al., 2000; Brent et al., 1998; Calam et al., 2002; Cobham et al., 1998; Hemphill \& Littlefield, 2006; Kazdin, 1995; Kazdin \& Wassel, 1998; Reyno \& McGrath, 2006; Webster-Stratton \& Hammond, 1990). Thus, it is not surprising that the significant relationship found in this study serves to further support the empirical observation that parent functioning is associated with youth outcomes in therapy opposed to youth symptoms predicting changes in parent functioning.

Several general aspects of this study warrant particular emphasis. A significant strength of this study is its context within a traditional community mental health setting. As discussed previously, one of the most notable deficiencies in child psychotherapy research has been the relative lack of studies examining treatment outcomes in "real-world" community settings, which is particularly problematic given the important differences between how psychotherapy is conducted in controlled clinical trials versus traditional outpatient clinics (Garland, Hurlburt \& Hawley, 2006; Kazdin, 2003; Weersing \& Weisz, 2002; Weisz et al., 2005). Studies such as this one are important as they help lend evidence to theory that has largely been established in controlled clinical trials (Garland et al, 2006; Kazdin, 2003; Weersing \& Weisz, 2002; Weisz \& Kazdin, 2003). This study is then particularly useful in supporting previous research findings examining the link between parent symptomatology and functioning and youth psychotherapy outcomes. Further, this study suggests that even in traditional community settings, parents indirectly benefit from their children receiving therapy, providing additional evidence that the scope of youth psychotherapy has been largely overlooked (Kazdin \& Wassel, 2000). 
Another strength of this study lies in the use of Hierarchical Linear Modeling (HLM). Inherent in these analyses are the benefits of using all available data points and the tolerance of missing data, particularly relevant given the study context where missing data were anticipated and observed. Unlike "typical” pre-post analyses, these sophisticated mixed model analyses allowed me to examine the shape and rate of change as well as covariates of change (Laurenceau et al, 2007; Singer \& Willett, 2003). Further, hierarchical linear modeling revealed how changes in parent functioning related to changes in youth symptoms over the course of treatment. Although the absence of a control group did not allow for a direct test of mediation, these analyses suggest, as mentioned previously, that parent social role performance and overall symptomatology may play a mediating role and warrant additional study.

\section{Limitations}

This study's results should be considered within the context of some notable limitations. One methodological concern is that reports of child psychopathology were obtained from the parent. Parent report may have led to an underestimation of internalizing problems as parents are better reporters of child externalizing problems. Further, as expected, some parents $(111,47 \%)$ in this study showed elevations on the OQ-45 indicating symptoms of psychopathology. Research regarding the accuracy with which parents who evidence psychopathology rate the psychological and behavioral symptoms of their children is mixed in its conclusions. Richters (1992) reviewed 22 studies from 1967 to 1990 looking at how accurately depressed mothers rated their children's behavioral problems. Seventeen of these articles supported the idea that depressed mothers report higher levels of behavioral problems, while five reported the contrary. However, the articles describing evidence that depressed mothers report higher levels of behavioral problems in their children did not meet necessary and sufficient criteria for demonstrating distorted ratings. The 
five articles that did not support the idea that depressed mothers inflate the observed behavior problems in their children were well conducted and used appropriate metrics. These findings led the author to conclude stronger evidence that depressed mothers are not distorted or inaccurate raters of their children’s behavior problems (Richters, 1992). Despite this evidence that parental psychopathology does not necessarily inflate ratings of child psychopathology, as well as decent correlations between the parent report version of the Y-OQ and the Y-OQ-SR, the findings based solely on parent rating should be interpreted with some caution.

Another limitation in this study is that participants were not formally assessed for diagnoses. Thus, while the diagnoses were diverse and varied as one would expect to find in a traditional clinic setting, the reliability of these diagnoses could not be estimated as they were subjective judgments made by the service providers giving treatment. This study examined child symptoms as measured by the Y-OQ opposed to diagnosis, but this is an important consideration given that specific psychological disorders likely have different impact on the children and estimates of treatment trajectories. For example, a child with severe ADHD will likely require psychotherapy that is greater in dose and longer in duration than a child with a simple phobia. Further, one would expect different diagnoses to differentially affect parent functioning. For example, a parent of a conduct disordered child might experience greater distress and symptomatology compared to parent who has a child with general anxiety. While severity of symptoms and overall general distress was examined, I had no means of differentially examining the ways in which diagnoses affected outcomes in this study because no formal assessment of diagnoses was provided.

Further, treatment implementation was not controlled for. That is, therapists varied in psychotherapy orientation and may or may not have followed specific evidence based treatment 
protocols depending on presenting problems. In general, treatment followed a family systems orientation and involved individual and family psychotherapy. Specific emphases included changing family interactional patterns, teaching problem-solving and decision-making strategies, and exploring circumstances that led to current problems. However, therapy was catered based on child presenting symptoms and clinician preference and subsequently it was not possible to know in what ways this may have affected outcomes.

Another limitation is lack of information regarding how often parents attended sessions with or without their children. Due to a family systems emphasis at the clinic where data was collected, it is known that parents were often involved in the psychotherapy treatment. However, it is not known the degree of involvement nor what parent participation looked like. For example, it is not known if parent distress was a target of psychotherapy or if sessions involving parents were largely focused on teaching parenting skills. The degree of parent involvement and the type of interventions targeted at parents could have influenced treatment outcomes but this lack of information does not allow for conclusions to be drawn in this regard.

It is also unknown whether parents were conjointly receiving individual or couples therapy themselves. This lack of information did not allow for conclusions regarding the ways in which parent treatment may have affected outcomes in this study.

Finally, this study only used the Y-OQ and OQ-45 as measures of child and parent symptomatology respectively. While these measures are comprehensive assessments of overall distress and symptomatology, additional measures or methods of assessment would be ideal to address the limitations inherent in a mono-method approach. However, as discussed previously, these measures show good reliability and validity in assessing overall psychological distress and sensitivity to change and are used in a variety of clinical settings. 


\section{Study Applications and Future Directions}

This study underscores the importance of attending to parent functioning both in the assessment and treatment phase for youth presenting for mental health services in traditional community settings. Predictors of treatment success or failure such as overall parent symptomatology, dissatisfaction in interpersonal relationships and social role performance, can

help clinicians be aware of clients that may be less likely to respond to treatment. For example, a mother who reports significant symptomatology and little satisfaction and sense of adequacy in her role as a parent may be less able to implement home-based interventions introduced within therapy, subsequently minimizing youth treatment gains and maintenance outside of therapy. Based on these findings, therapists may want to include therapy sessions directly targeted at assessing and alleviating parent symptoms and improving parent interpersonal relationships and satisfaction in their social roles, as well as making referrals for parents to receive services themselves where appropriate.

This study further provides evidence that psychotherapy for youth in a traditional community setting indirectly benefits parents. This is an important finding, indicating that psychotherapy for youth may provide broader reaching effects than previously theorized (Kazdin \& Wassel, 2000). Such findings have important implications as improvement in parent symptoms and functioning may help to facilitate and maintain therapeutic progress in youth (e.g. Kazdin, 2004). This is particularly relevant given the traditional outpatient setting in which this study was conducted, where reviewed research in similar settings demonstrate minimal therapeutic gains (Garland et al., 2005). Thus, these findings not only indicate significant therapeutic gains that extend to the parents of youth being treated, but also suggest more 
optimistic outcomes in regards to the maintenance of therapeutic gains due to improved family functioning.

This study provides a useful foundation for subsequent research on the association of parent functioning and youth psychotherapy outcomes as well as how youth psychotherapy indirectly benefits parents; however, more research is needed to further elucidate this complex relationship. For example, through the use of treatment and control groups, questions could be addressed regarding mediation and the directionality of effects in the association between changes in parent functioning and changes in youth symptoms over the course of treatment. In addition, very little attention has been devoted to examining the relation between parent social role performance and youth psychotherapy outcomes. Further research is needed to highlight this potentially important association; subsequent research will be needed to further examine the specific aspects of parent social role performance that are most strongly associated with youth symptoms and outcomes. Finally, it may be advantageous if future research examined whether providing clinicians and parents with specific feedback on parent functioning in the context of youth treatment would facilitate treatment gains and decrease the frequency of negative outcomes. 


\section{References}

Achenbach, T. M. (1991). Manual for the Child Behavior Checklist / 4-18 and 1991 Profile. Burlington, VT: University of Vermont Department of Psychiatry.

Anselmi, L., Piccini, C. A., Barros, F. C. \& Lopes, R. S. (2004). Psychosocial determinants of behavior problems in Brazilian preschool children. Journal of Child Psychology and Psychiatry, 45, 779-788.

Baron, R. M., \& Kenny, D. A. (1986). The moderator-mediator variable distinction in social psychological research: Conceptual, strategic, and statistical considerations. Journal of Personality and Social Psychology, 51, 1173-1182.

Barret, P. M., Dadds, M. R., \& Rapee, R. M. (1996). Family treatment of childhood anxiety: A controlled trial. Journal of Consulting and Clinical Psychology, 64, 333-342.

Berman S. L., Weems, C. F., Silverman, W. K. \& Kurtines, W. M. (2000). Predictors of outcome in exposure-based cognitive and behavioral treatments for phobic and anxiety disorders in children. Behavior Therapy 31, 713-731.

Berrett, K. M. (1999). Youth Outcome Questionnaire: Item sensitivity to change. Unpublished doctoral dissertation, Brigham Young University, Provo, UT.

Blader, J. C. (2006). Which family factors predict children’s externalizing behaviors following discharge from psychiatric inpatient treatment. Journal of Child Psychology and Psychiatry, 47, 1133-1142.

Brent, D. \& Kolko, D. (1998). Psychotherapy: Definitions, Mechanisms of Action, and Relationship to Etiological Models. Journal of Abnormal Child Psychology, 26, 17-25.

Brent, D. A., Kolko, D. J., Birmaher, B., Baugher, M., Bridge, J., Roth, C., \& Holder, D. (1998). Predictors of treatment efficacy in a clinical trial of three psychosocial 
treatments for adolescent depression. Journal of the American Academy of Child and Adolescent Psychiatry, 37, 906-914.

Bryk, A. S., \& Raudenbush, S. W. (1987). Application of hierarchical linear models to assessing change. Psychological Bulletin, 101, 147-158.

Burlingame, G. M., Wells, M. G., Hoag, M., Hope, C., Nebeker, S., Konkel, K., et al. (1996). Administration and scoring manual for the Youth Outcome Questionnaire (Y-OQ.1). Stevenson, MD: American Professional Credentialing Services.

Burlingame, G. M., Mosier, J. I., Wells, M. G., Atkin, Q. G., Lambert, M. J., Whoolery, M., et al. (2001). Tracking the influence of mental health treatment: The development of the Youth Outcome Questionnaire. Clinical Psychology and Psychotherapy, 8, 315-334.

Burlingame, G. M., Wells, M. G., Lambert, M. J., \& Cox, J. C. (2004). Youth Outcome Questionnaire (Y-OQ). In M. Maruish (Ed.) The use of psychological tests for treatment planning and outcome assessment ( $3^{\text {rd }}$ ed.). New Jersey: Lawrence Erlbaum Associates.

Calam, R., Bolton, C. \& Roberts, J. (2002). Maternal expressed emotion, attributions and depression and entry into therapy for children with behavior problems. British Journal of Clinical Psychology, 41, 213-216.

Calam, R. Bolton, C., Barrowclough, C., \& Roberts, J. (2002). Maternal expressed emotion and clinician ratings of emotional maltreatment potential. Child Abuse and Neglect, 26, 11011106.

Clarke, G., Hops, H., Lewinsohn, P., Andrews, J., Seeley, J., \& Williams, J. (1992). Cognitive-behavioral group treatment of adolescent depression: Prediction of outcome. Behavior Therapy, 23, 341-354. 
Cobham, V. E., Dadds, M. R., Spence, S. H. (1998). The role of parental anxiety in the treatment of childhood anxiety. Journal of Consulting and Clinical Psychology, 66, 893-905.

Conners, C. K. Conners Rating Scales. In M. E. Maruish (Ed). The use of psychological testing for treatment planning and outcome assessment. (pp. 550-578). New Jersey: Lawrence Erlbaum Associates, Inc.

Costa N., Dalton, R., Pellerin, K. \& Weems, C. (2006). Parenting stress and childhood psychopathology: An examination of specificity to internalizing and externalizing symptoms. Journal of Psychopathology and Behavioral Assessment, 28, 113-122.

Cox, M. J., Blair, P. \& Harter, K. (2001). Interparental conflict and parent-child relationships. In J. H. Grych \& F. D. Fincham (Eds). Interparental conflict and child development: Theory, research, and applications. (pp. 249-272). New York: Cambridge University Press.

Crnic, K. A., Gaze, C. \& Hoffman, C. (2005). Cumulative parenting stress across the preschool period: relations to maternal parenting and child behaviour at age 5. Infant and Child Development, 14, 117-1132.

Cummings, E. M. \& Davies, P. T. (2002). Effects of marital conflict on children: Recent advances and emerging themes in process-oriented research. Journal of Child Psychology and Psychiatry, 43, 31-63.

Deater-Deckard, K. (1998). Parenting stress and child adjustment: some old hypotheses and new questions. Clinical Psychology: Science and Practice, 5, 314-332.

Downey, G. \& Coyne, J. (1990). Children of depressed parents: An integrative review. Psychological Bulletin, 108, 50-76. 
Durlak, J. A., Fuhrman, T., \& Lampman, C. (1991). Effectiveness of cognitive-behavioral therapy for maladapting children: A meta-analysis. Psychological Bulletin, 110, 204-214.

Erel, O. \& Burman, B. (1995). Interrelatedness of martial relations and parent-child relations: A meta-analytic review. Psychological Bulletin, 118, 108-132.

Finch, A. E., Lambert, M. J., \& Schaalje, B. G. (2001). Psychotherapy quality control: The statistical generation of expected recovery curves for integration into an early warning system. Clinical Psychology and Psychotherapy, 8, 231-242.

Forehand,R., Wierson, M., Thomas, A. M. \& Fauber, R. (1991). A short-term longitudinal examination of young adolescent functioning following divorce: The role of family factors. Journal of Abnormal Child Psychology, 19, 97-111.

Gallimore, M. \& Kurdek, L. (1992). Parent depression and parent authoritative discipline as correlates of young adolescents’ depression. Journal of Early Adolescence, 12, 187-196.

Garcia, J. A. \& Weisz, J. R. (2002) When youth mental health care stops: Therapeutic relationship problems and other reasons for ending youth outpatient treatment. Journal of Consulting and Clinical Psychology, 70, 439-443.

Gardner, F., Burton, J., \& Klimes, I. (2006). Randomised controlled trial of a parenting intervention in the voluntary sector for reducing child conduct problems: outcomes and mechanisms of change. Journal of Child Psychology and Psychiatry, 47, 1123-1132.

Garland, A. F., Hurlburt, M. S., Hawley, K. M. (2006). Examining psychotherapy processes in a services research context. Clinical Psychology: Science and Practice, 31, 30-46.

Grych, J. H. \& Fincham, F. D. (1990). Marital conflict and children’s adjustment: A cognitive contextual framework. Psychological Bulletin, 108, 267-290. 
Hammen, C. (1990). The family-environmental context of depression: A perspective on children’s risk. In D. Cicchetti \& S. I. Toth (Eds). Rochester Symposium on Developmental Psychopathology, Vol IV: Developmental Perspectives on Depression (pp. 1-40). New York: University of Rochester Press.

Hammen, C., Brennan, P. A., Shih, J. H. (2004). Family discord and stress predictors of depression and other disorders in adolescent children of depressed and nondepressed women. Journal of the American Academy of Child and Adolescent Psychiatry, 43, 9941002.

Hansen, N. B., Lambert, M. J., \& Forman, E. M. (2002). The psychotherapy dose-response effect and its implications for treatment delivery services. Clinical Psychology Science \& Practice, 9, 329-343.

Harnish, J., Dodge, K. \& Valente, E. (1995). Mother-child interaction quality as a partial mediator of the roles of maternal depressive symptomatology and socioeconomic status in the development of child behavior problems. Child Development, 66, 739-753.

Harold, G. T. \& Conger, R. D. (1997). Marital conflict and adolescent distress: The role of adolescent awareness. Child Development, 68, 333-350.

Harold, G. T., Shelton, K. H., Goeke-Morey, M. C. \& Cummings, E. M. (2004). Marital conflict, child emotional security about family relationships and child adjustment. Social Development, 13. 350-376.

Hemphill, S. A. \& Littlefield, L. (2006) Child and family predictors of therapy outcome for children with behavioral and emotional problems. Child Psychiatry and Human Development, 36, 329-349. 
Holmbeck, G. N. (1997). Toward terminological, conceptual, and statistical clarity in the study of mediators and moderators: Examples from the child-clinical and pediatric psychology literatures. Journal of Consulting and Clinical Psychology, 65, 599-610.

Howard, K. I., Moras, K., Brill, P. L., Martinovich, Z., \& Lutz, W. (1996). Evaluation of psychotherapy: Efficacy, effectiveness, and patient progress. American Psychologist, 51, 1059-1064.

Jacobson, N. S., \& Truax, P. (1991). Clinical significance: A statistical approach to defining meaningful change in psychotherapy research. Journal of Consulting and Clinical Psychology, 59, 12-19.

Jaser, S. S., Langrock, A. M., Keller, G., Merchant, M. J., Benson, M. A., Reeslund, K., Champion, J. E. \& Compas, B. E. (2005). Coping with the stress of parental depression II: Adolescent and parent reports of coping and adjustment. Journal of Clinical Child and Adolescent Psychology, 34, 193-205.

Jayson, D., Wood, A., Kroll, L., Fraser, J., \& Harrington, R. (1998). Which depressed patients respond to cognitive-behavior treatment? Journal of the American Academy of Child and Adolescent Psychiatry, 37, 35-39.

Kazdin, A. E. (1991) Effectiveness of psychotherapy with children and adolescents. Journal of Consulting and Clinical Psychology, 59, 785-798.

Kazdin, A. E. (1994). Psychotherapy for children and adolescents. In A. Bergin \& S. Garfield (Eds.), Handbook of psychotherapy and behavior change ( $4^{\text {th }}$ ed.), (pp. 543-594). New York: John Wiley \& Sons, Inc. 
Kazdin, A. E. (1995). Child, Parent and Family Dysfunction as Predictors of Outcome in Cognitive-Behavioral Treatment of Antisocial Children. Behavior Research and Therapy, 33, 271-281.

Kazdin, A. E. (2003). Psychotherapy for children and adolescents. Annual Reviews Psychology, 54, 253-276.

Kazdin, A. E. (2004). Psychotherapy for children and adolescents. In M. Lambert (Ed). Bergin and Garfield's handbook of psychotherapy and behavior change (5th ed.), (pp. 543-589). New York: John Wiley \& Sons, Inc.

Kazdin, A. E. (2006). Mechanisms of change in psychotherapy: Advances, breakthroughs, and cutting-edge research (do not yet exist). In R. Bootzin \& P. McKnight (Eds.) Strengthening research methodology: Psychological measurement and evaluation (pp.77 -101). Washington, DC: American Psychological Association.

Kazdin, A. E. \& Crowley M. (1997). Moderators of Treatment Outcome in Cognitively Based Treatment of Antisocial Children. Cognitive Therapy and Research, 21, 185207.

Kazdin, A. E., Holland, L. \& Crowley, M. J. (1997). Family experience of barriers to treatment and premature termination from child therapy. Journal of Consulting and Clinical Psychology, 65, 453-463.

Kazdin A. E. \& Kendall P. C. (1998). Current progress and future plans for developing effective treatments: Comments and perspectives. Journal of Clinical Child Psychology 27, 217 $-226$. 
Kazdin, A. E. \& Mazurick, J. L. (1994). Dropping out of child psychotherapy: Distinguishing early and late dropouts over the course of treatment. Journal of Consulting and Clinical Psychology, 62, 1069-1074.

Kazdin, A. E. \& Nock, M. K. (2003). Delineating mechanisms of change in child and adolescent therapy: methodological issues and research recommendations. Journal of Child Psychology and Psychiatry 44, 1116-1129.

Kazdin, A. E. \& Wassel, G (1998) Treatment completion and therapeutic change among children referred for outpatient therapy. Professional Psychology;Research and Practice 29, 332 $-340$.

Kazdin, A. E. \& Wassel, G. (2000). Therapeutic changes in children, parents, and families resulting from treatment of children with conduct problems. Journal of the American Academy of Child and Adolescent Psychiatry 39, 414-420.

Kendall, P. C. (1994). Treating anxiety disorders in children: Results of a randomized clinical trial. Journal of Consulting and Clinical Psychology, 62, 100-110.

Kendall P. C., Holmbeck, G \& Verduin, T. (2004) Methodology, design and evaluation in psychotherapy research. In M. J. Lambert (Ed.) Bergin and Garfield's handbook of psychotherapy and behavior change, (5 ${ }^{\text {th }}$ ed),. (pp.16-44). New York: John Wiley \& Sons, Inc.

Krishnakumar, A., Buehler, C. \& Barber, B. K. (2003) Youth perceptions of interpersonal conflict, ineffective parenting, and youth problem behaviors in European-American and African-American families. Journal of social and personal relationships, 20, 239-260. 
Lambert, M. J. (1998, August). Patterns of patient improvement: Implications of research for treatment planning and responsible social policy. Paper presented at the Seventeenth World Conference of Psychotherapy, Warsaw, Poland.

Lambert, M. J., Whipple, J. L., Bishop, M. J., Vermeersch, D. A., Gray, G. V., \& Finch, A. E. (2002). Comparison of empirically-derived and rationally-derived methods for identifying patients at risk for treatment failure. Clinical Psychology and Psychotherapy, 9, 149-164.

Lambert, M. J., Morton, J. J., Hatfield, D., Harmon, C., Hamilton, S., Reid, R. C., Shimokawa, K., Christopherson, C. \& Burlingame, G. M.. (2004). Administration and scoring manual for the outcome questionnaire (OQ 45.2). Wilmington, DE: American Professional Credentialing Services.

Laurenceau, J. P., Hayes, A. M., \& Feldman, G. C. (2007). Some methodological and statistical issues in the study of change processes in psychotherapy. Clinical Psychology Review, 27, 682-695.

Li, S. A. (1997) Effects of interpersonal conflict and loss events on Mexican-American and Anglo-American adolescent adjustment. Dissertation Abstracts International: Section B: The Sciences and Engineering, 58(6-B), 3320.

Lutz, W., Stulz, N., Smart, D.W., \& Lambert, M. J. (2007). Patterns of early change in outpatient therapy. Journal of Clinical Psychology and Psychotherapy, 36, 93-104.

Marchand, J. \& Hock, E. (1998). The relation of problem behaviors in preschool children to depressive symptoms in mothers and fathers. The Journal of Genetic Psychology, 159, 353-366. 
Mash, E.J. \& Dozois, D.J. (2003) Child psychopathology: A developmental-systems perspective. In Mash, E.J. \& Barkley R.A. (Eds.) Child Psychopathology (pp. 3-75). New York: Guilford Press.

McBurnett, K. Pfiffner, L., Capasso, L., Lahey, B., \& Loeber, R. (1997). Children’s aggression and DSM-III-R symptoms predicted by parent psychopathology, parenting practices, cortisol and SES. In A. Raine, P. Brenna, D. Farrington \& S. Mednick (Eds.), Biosocial bases of violence (pp.345-348). New York: Plenum Press.

Mosier, J., Burlingame, G., Wells, G., Ferre, R., Latkowski, M., Johansen, J., et al. (2001).In-home, family centered psychiatric treatment for high-risk children and youth. Children's Services: Social Policy, Research, and Practice, 4, 51-68.

Mueller, R. M., Lambert, M. J., \& Burlingame, G. M. (1998). Construct validity of the Outcome Questionnaire: A Confirmatory factor analysis. Journal of Personality Assessment, 70, 248 $-262$.

Nye, C.L., Zucker, R.A., \& Fitzgerald, H.E. (1995). Early intervention in the path to alcohol problems through conduct problems: Treatment involvement and child behavior change. Journal of Consulting and Clinical Psychology, 63, 831-840.

Papp, L. M., Cummings, E. M. \& Goeke-Morey, M. C. (2005). Parent psychological distress, parent-child relationship qualities and child adjustment: Direct, mediating and reciprocal pathways. Parenting: Science and Practice, 5, 259-283.

Pina, A.A., Silverman, W.K., Weems, C.F., Kurtines, W.M. \& Goldman, M.L. (2003). A comparison of completers and noncompleters of exposure-based cognitive behavioral treatment for phobic and anxiety disorders in youth. Journal of Consulting and Clinical Psychology, 71, 701-705. 
Reyno, S. M. \& P. J. McGrath (2006). Predictors of parent training efficacy for child externalizing behavior problems-a meta-analytic review. Journal of Child Psychology and Psychology, 47, 99-111.

Richters, J.E. (1992). Depressed mothers as informants about their children: A critical review of the evidence for distortion. Psychological Bulletin, 112, 485-499.

Ridge, N. W., Warren, J. S., Burlingame, G. M., Wells, G. M. (2007). The reliability, concurrent validity, and factor structure of the Youth Outcome Questionnaire Self-Report. Dissertation Abstracts International: B. The Sciences and Engineering, 68, 3409.

Robinson, K. (2000). Outcomes of a partial-day treatment program for referred children. Child and Youth Care Forum, 29, 127-137.

Russel, K. (2003). An assessment of outcomes in outdoor behavior healthcare treatment. Child and Youth Care Forum 32, 355-381.

Singer, J. D. \& Willettt, J. B. (2003). Applied longitudinal data analysis: Modeling change and event occurrence. New York: Oxford University Press.

Southam-Gerow, M.A., Kendall, P.C., \& Weersing, V.R. (2001). Examining outcome variability: Correlates of treatment response in a child and adolescent anxiety clinic. Journal of Clinical Child Psychology, 30, 422-436.

Spielmans, G.I., Masters, K.S., \& Lambert, M. J. (2006). A comparison of rationale versus empirical methods in the prediction of psychotherapy outcome. Clinical Psychology and Psychotherapy, 13, 202-214.

Warren, J.S., Nelson, P.L. \& Burlingame, G.M. (In Press). Identifying youth at risk for treatment failure in outpatient community mental health services. Journal of Child and Family Studies. 
Webster-Stratton, C. \& Hammond, M. (1990). Predictors of treatment outcome in parent training for families with problem children. Behavior Therapy, 21, 319-337.

Weersing, V. R. \& Weisz, J. R. (2002). Mechanisms of action in youth psychotherapy. Journal of Child Psychology and Psychiatry 43, 3-29.

Weisz, J. R., Jensen, A. L., \& McLeod, B. D. (2005). Development and dissemination of child and adolescent psychotherapies: Milestones, methods, and a new deployment-focused model. Psychosocial Treatments for Child and Adolescent Disorders: Empirically Based Strategies for Clinical Practice (2 ${ }^{\text {nd }}$ ed), 9-39.

Weisz, J. R. \& Kazdin, A. E. (2003). Concluding thoughts: present and future of evidence-based psychotherapies for children and adolescents. In A. E. Kazdin \& J. R. Weisz (Eds.), Evidence-based psychotherapies for children and adolescents, (pp.439-451). New York: Guilford Press.

Wells, M. G., Burlingame, G. M., \& Rose, P. M. (2003). Youth Outcome Questionnaire Self report. Wilmington, DE: American Professional Credentialing Services.

Zimet, D. M. \& Jacob, T. (2001). Influences of marital conflict on child adjustment: Review of theory and research. Clinical Child and Family Psychology Review, 4, 319-335. 